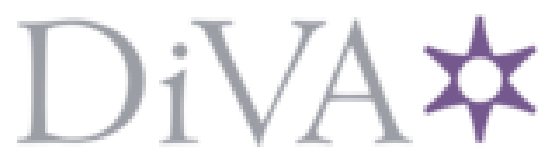

http://www.diva-portal.org

This is the published version of a paper presented at 32nd AIAA Applied Aerodynamic Conference.

Citation for the original published paper:

Mengmeng, Z. (2014)

Collaborative Aircraft Design Methodology using ADAS linked to CEASIOM.

In: 32nd AIAA Applied Aerodynamic Conference

32nd AIAA Applied Aerodynamic Conference

http://dx.doi.org/10.2514/6.2014-2012

N.B. When citing this work, cite the original published paper.

Permanent link to this version:

http://urn.kb.se/resolve?urn=urn:nbn:se:kth:diva-168164 


\title{
Collaborative Aircraft Design Methodology using ADAS Linked to CEASIOM
}

\author{
M. Zhang*, A. Rizzi†, \\ Royal Institute of Technology (KTH), 10044 Stockholm, Sweden \\ F. Nicolosi $;$ A. De Marco \\ University of Naples Federico II, Italy
}

\begin{abstract}
The aircraft design stages, conceptual and preliminary, are necessarily collaborative by their very nature. An example design carried out in this paper brings the collaborative aspects of design to life by two academic groups, one in Naples and one in Stockholm, using their own tools ADAS and CEASIOM, working respectively on conceptual and preliminary design. The ADAS tool is primarily empirically-based design methodology, and the CEASIOM tool is primarily physics-based design methodology. The example chosen is a FAR-23 compliant 16-seat twin turboprop aircraft. The high-wing configuration resulting from the ADAS conceptual design is the down-selected to CEASIOM where a water-tight model of the geometry is constructed, a volume grid is generated and 16 flight conditions are simulated by solutions of the Euler equations, some with propeller off, and others with propeller in order to judge the effect of the propeller wash over the main wing and horizontal tail surface. Detailed comparisons between ADAS results and CEASIOM results for stability \& control characteristics are carried out. In general there is reasonable agreement between the two sets, considering that the empiricisms in ADAS account for viscous effects where as the CEASIOM are purely inviscid (but nonlinear). The largest discrepancy appears in the pitching moment contribution from the horizontal tail, and various explanations for this are suggested, including possible effects of the main wing downwash and wake on the tail.
\end{abstract}

\section{Introduction \& Overview}

Figure 1 spells out the details in the early steps of aircraft design for the definition of the configuration, as carried out as an industrial endeavor (as opposed to an academic one). It illustrates two design loops in the conceptual design phase that follow the first-guess sizing (usually done by a spread-sheet or similar tool) to obtain the initial layout of the configuration. The first one, the pre-design loop, is aimed at establishing a very quick (time-scale can be from one to a few weeks) yet technically consistent-sized configuration with a predicted performance. The second one, the concept-design loop, is a protracted and labor intensive effort involving more advanced first-order trade studies to produce a refinement in defining the minimum goals of a candidate project. At the end of the conceptual design phase all the design layouts will have been analyzed, and the best one, or possibly two, designs will be down-selected to the preliminary design phase. During the preliminary definition, project design is still undergoing a somewhat fluid process and indeed warrants some element of generalist-type thinking, but the minimum goals of the project have already been established during the conceptual definition phase, and so the aim here is to meet these targets using methods with higher order than those used during the conceptual definition phase in order sharpen the focus to achieve refinements in the targets. Furthermore, the participants in this working group are mostly genuine specialists in each respective discipline. Figure 1 indicates the way in which data, or information, is passed between specialist groups during the design process. The specialist groups must consider the level of advanced technology to be adopted together with all of the other active constraints on the design. The data

*Ph.D. student, AIAA member, e-mail: mzha@kth.se

$\dagger$ Professor, AIAA fellow, e-mail: rizzi@kth.se

$¥$ Associate Professor, AIAA member, e-mail: fabrnico@unina.it

$\S$ Assistant Professor, AIAA member, e-mail: agostino.demarco@unina.it 
flow lines indicate how the technology areas influence the aircraft configuration through its performance. The specialist departments/offices provide the input data to the project designers who then coordinate a systematic search to find the optimum configuration (for example the optimum wing shape for the mission, e.g. cruise) and to settle disputes between conflicting specialist opinions.

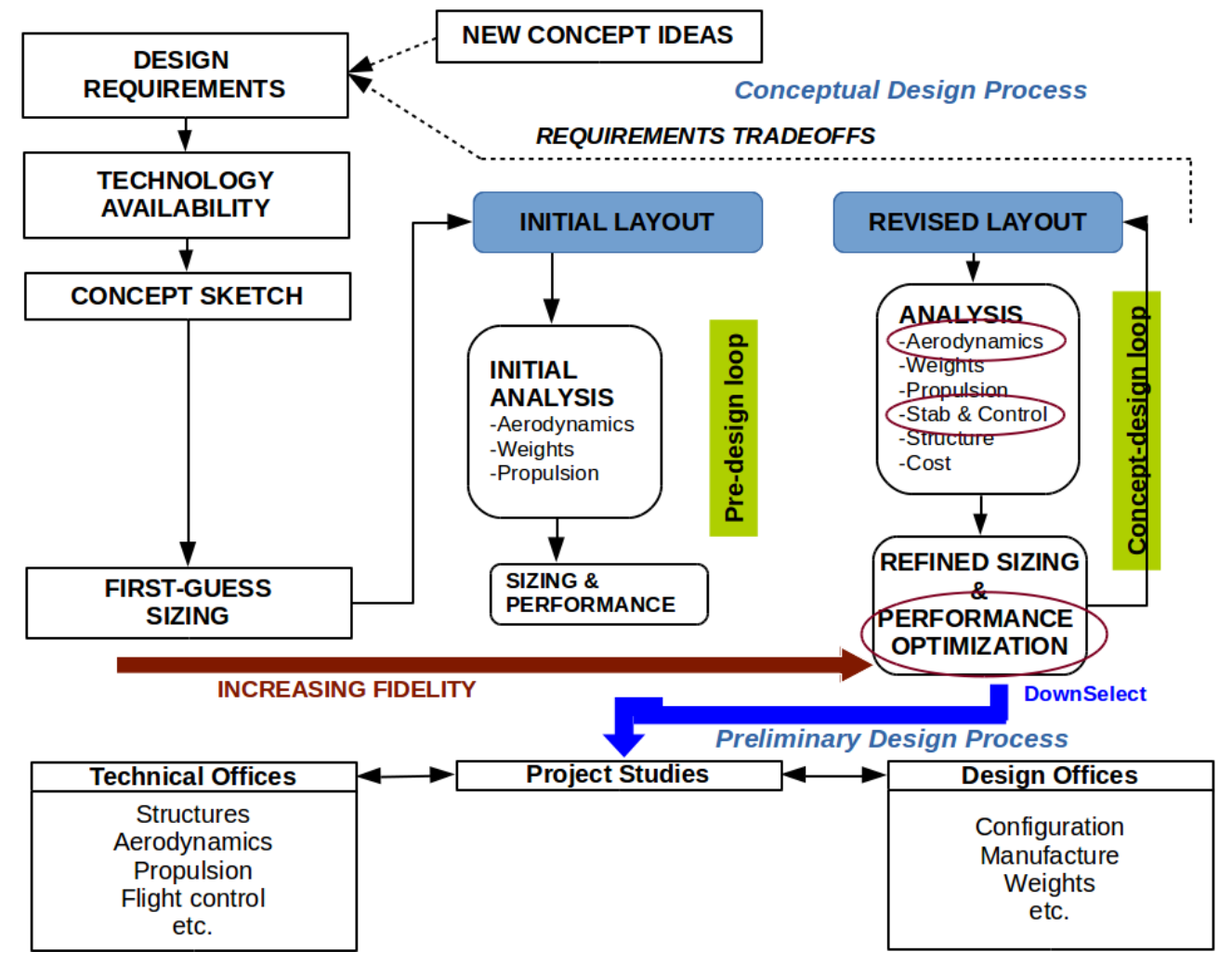

Figure 1. The two design loops in the conceptual design phase process and the down-select to project study in preliminary design

There exists today a good deal of inefficiencies in interactions between all these various groups incurred as the loss of, or incompatibility of, data which it is transmitted between the groups/stages, with the result that the data must be re-generated or re-worked in order to be useful at the next design stage. CAD modeling and grid generation are two cases in point of such inefficiency. Typically geometrical modeling in the conceptual stage does not work with trimmed surfaces, water-tight models or what could be called a 'meshable' geometry. Thus when such a model arrives at the preliminary design stage, substantial work in CAD-repair must be undertaken before useful work can begin. Better collaboration between the tools at the right time can help reduce this re-work.

The thesis of this paper is that there are several and different tools in the tool-chain to carry out this design process, and that the overall efficiency of the process is enhanced by increasing the collaboration between the different tools and by reducing the re-work required. This is not just an academic question, it is a real concern in industry, as can be seen by the the considerable efforts in the EU projects VIVACE and CRESCENDO have been putting into studying it, and devising possible ways to improve upon it, see for example. ${ }^{25}$ The goal of the paper is to illustrate the collaborative process, indicated in Fig 1 , by a concrete example - development of a FAR-23 16-seat aircraft. The two tools in question for this work are the Aircraft Design \& Analysis Software (ADAS) ${ }^{1}$ suite for conceptual design (pre-design \& concept design) and CEASIOM $^{16}$ for preliminary design. Apply first ADAS for the sizing procedure, and then CEASIOM to check and refine the data.

The paper is structured accordingly to develop this example. Section II presents a brief description of the two tools used, ADAS and CEASIOM, followed by Section III that details many of the steps taken, and the data generated, in the execution of ADAS - how it determines the sizing of all parts of the aircraft: wing, engine, flap, aileron, tails. It estimates the aerodynamic derivatives through semi-empirical procedures that are then checked in Section IV with physics-based analysis methods in CEASIOM. Section V presents a 


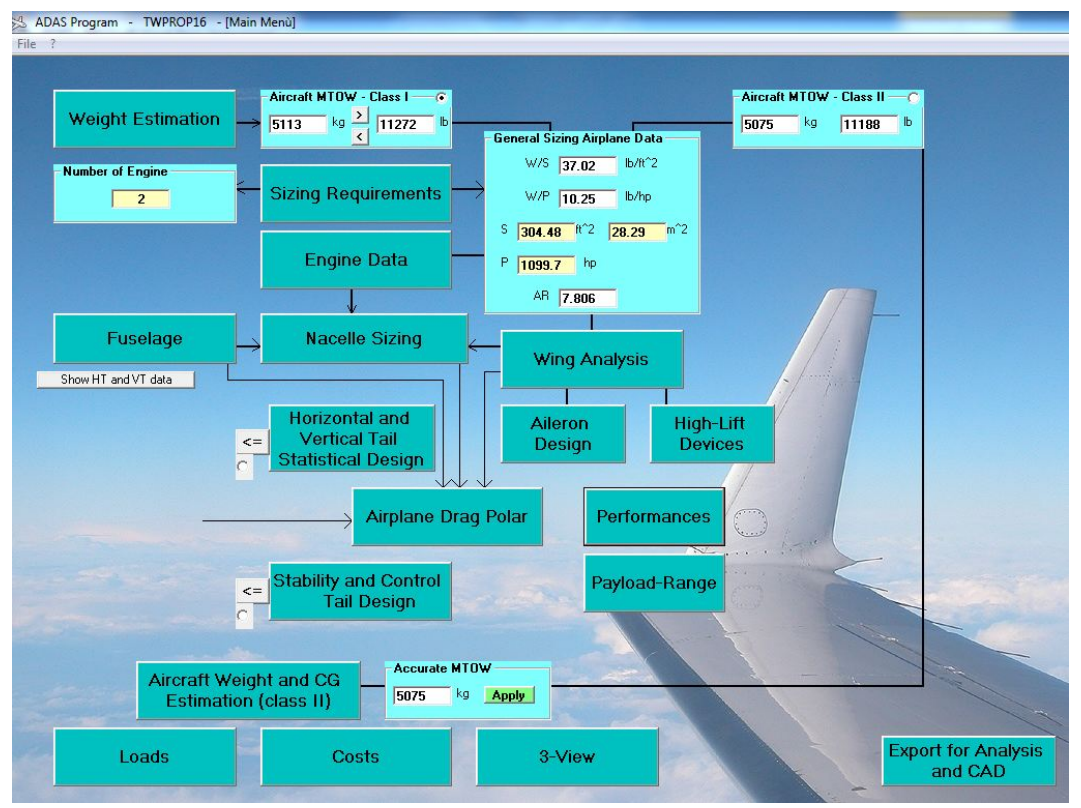

Figure 2. ADAS main GUI.

discussion on the comparisons and differences between the two sets of predictions, with evidence contradicting the idea that higher-fidelity always means more realism in the data. The truth is that when empiricisms hold, they are hard to beat, but it takes good engineering judgement to know when this is the case. Section $\mathrm{V}$ finishes with some conclusions and directions for future work.

\section{Brief Description of Tools}

\section{A. Aircraft Design \& Analysis Software (ADAS)}

The structure and functionalities of the ADAS (Aircraft Design and Analysis Software) tool have been presented by Nicolosi and Paduano. ${ }^{1,2}$ ADAS was originally intended as an educational tool but, after many improvements occurred to the internal code over the past years, ${ }^{5-9}$ it can be absolutely used as a research tool and has played a relevant role for some industrial applications as well. ${ }^{5,10-15}$

The estimation of all aircraft characteristics with ADAS is done by means of classical semi-empirical methods like those proposed by Roskam, ${ }^{30-32}$ ESDU ${ }^{36}$ or USAF Datcom. ${ }^{33-35}$ These methods are also integrated with more sophisticated approaches. For instance, ADAS implements Multhopp and Weissinger theories for straight and swept wings and the analysis of the main lifting surface relies on the numerical calculation of spanwise lift distribution. This feature allows the assessment of wing stall path.

One of the main goals of ADAS is to provide users with an intuitive and user friendly platform, that can be easily used both by practitioners and by students. The software comes with many visual helps and with many output graphs and figures, including 2D and 3D drawings of aircraft parts under analysis.

ADAS is split in several modules. The typical work session with the software starts with the module designed for the preliminary estimation of: aircraft weights (both empty weight and maximum take-off weight), required wing area, and engine thrust/power ratio under the specified design requirements. ${ }^{5}$ For this initial task all FAR/CS 23/25 performance requirements have been automatically implemented based on aircraft weight.

After the preliminary calculation step is set up according to the design requirements, the user then starts designing all aircraft parts. The main dialog window shown in Figure 2 allows to enter and carry out the design of each element of the configuration (fuselage, nacelle, wing, ailerons and flap, taiplanes). Each item in the GUI (Graphical User Interface) can be used as an independent subpart design module, but it is linked with the other modules if an airplane design activity is developed. Figure 3 shows a flowchart of typical design/analysis steps that the user can carry out in sequence in ADAS. 


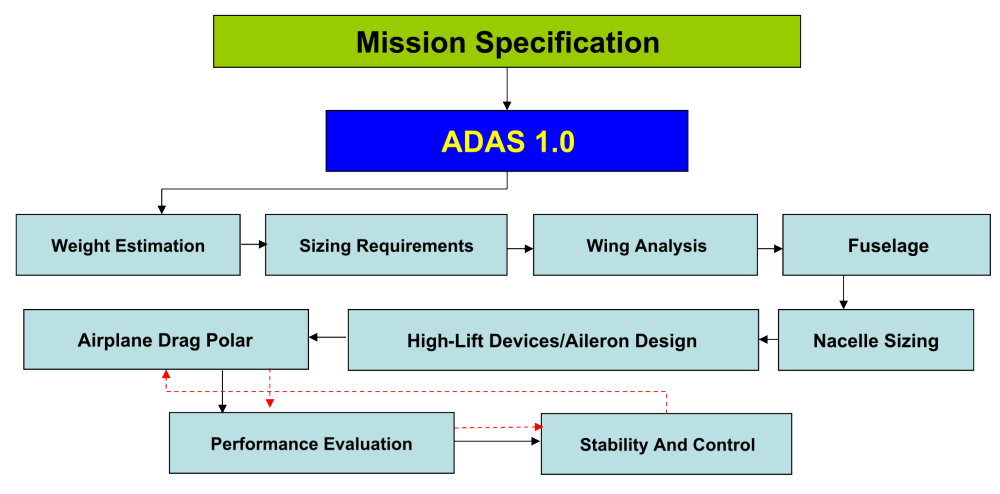

Figure 3. ADAS modules and flowchart of a typical design sequence.

Usually in aircraft design the construction of drag polar and main flight/ground performance estimation can be accomplished with a first preliminary sizing of tailplanes. The tailplane analysis modules in ADAS allow a detailed and accurate design of horizontal and vertical tailplane, performing also the aircraft stability and control analysis up to non-linear conditions.

The wing module allows the design of the wing and the calculation of all wing aerodynamics characteristics, including lift, moment and drag. The estimation of spanwise lift coefficient distribution and wing stall path are included in this module, giving the user the possibility to check the assumed wing twist and to make a preliminary estimation of wing bending moment.

The wing module is linked with the fuselage module to estimate the body longitudinal instability contribution which depends on wing induced upwash and downwash. The wing analysis is also coupled with the modules for high-lift and aileron analysis. The aileron analysis and design module allows the estimation of aircraft roll performances with the selected propulsive system.

The software has a module dedicated to the accurate estimation of aircraft drag polar and a module for aircraft flight and ground performances. Other very important modules deal with aircraft tail sizing and aircraft longitudinal and lateral-directional characteristics.

All the ADAS modules are conceived to be as user-friendly as possible and allow a fast calculation of all required information. A user experienced with ADAS is able to perform the preliminary design and analysis of a transport aircraft and produce a set of drawings of the designed configuration in less than 1 hour.

Other similar software are under development in other European Universities, e. g. PreSTo developed by Scholz, ${ }^{3}$ and such efforts demonstrate the relevance and the importance of these kind of tools, both in teaching and in some applied industrial cases. Jenkinson has written a book ${ }^{4}$ that enphasizes how important it is for students to have a hands-on approach in aircraft design education based on real-world examples.

\section{B. CEASIOM framework for variable-fidelity design}

CEASIOM, the Computerized Environment for Aircraft Synthesis and Integrated Optimization Methods, developed within the European 6th Framework Programme SimSAC (Simulating Aircraft Stability And Control Characteristics for Use in Conceptual Design), is a framework tool for conceptual aircraft design that integrates discipline-specific tools like: CAD \& mesh generation, CFD, structures, stability \& control analysis, etc., all for the purpose of early preliminary design. ${ }^{16}$ It is an ad hoc framework that offers possible ways to increase the concurrency and agility of the classical conceptual-preliminary process outlined in Fig. 1. Figure 4 presents an illustration of the CEASIOM software, showing aspects of its four core functions: geometry \& meshing, CFD, aeroelastics and S\&C (flight dynamics). Significant features developed and integrated in CEASIOM as modules are:

- Geometry module CPACScreator-sumo ${ }^{17,26}$ A customized geometry construction system coupled to automated surface and volume grid generators; Resulting model exported to CAD via IGES standard.

- Aerodynamic Model Builder AMB-CFD ${ }^{18}$ A complete toolbox of aerodynamic analysis methods ranging from the empirically based DATCOM to physics-based linear and non-linear CFD (Euler \& RANS) offering broad choice in fidelity: 


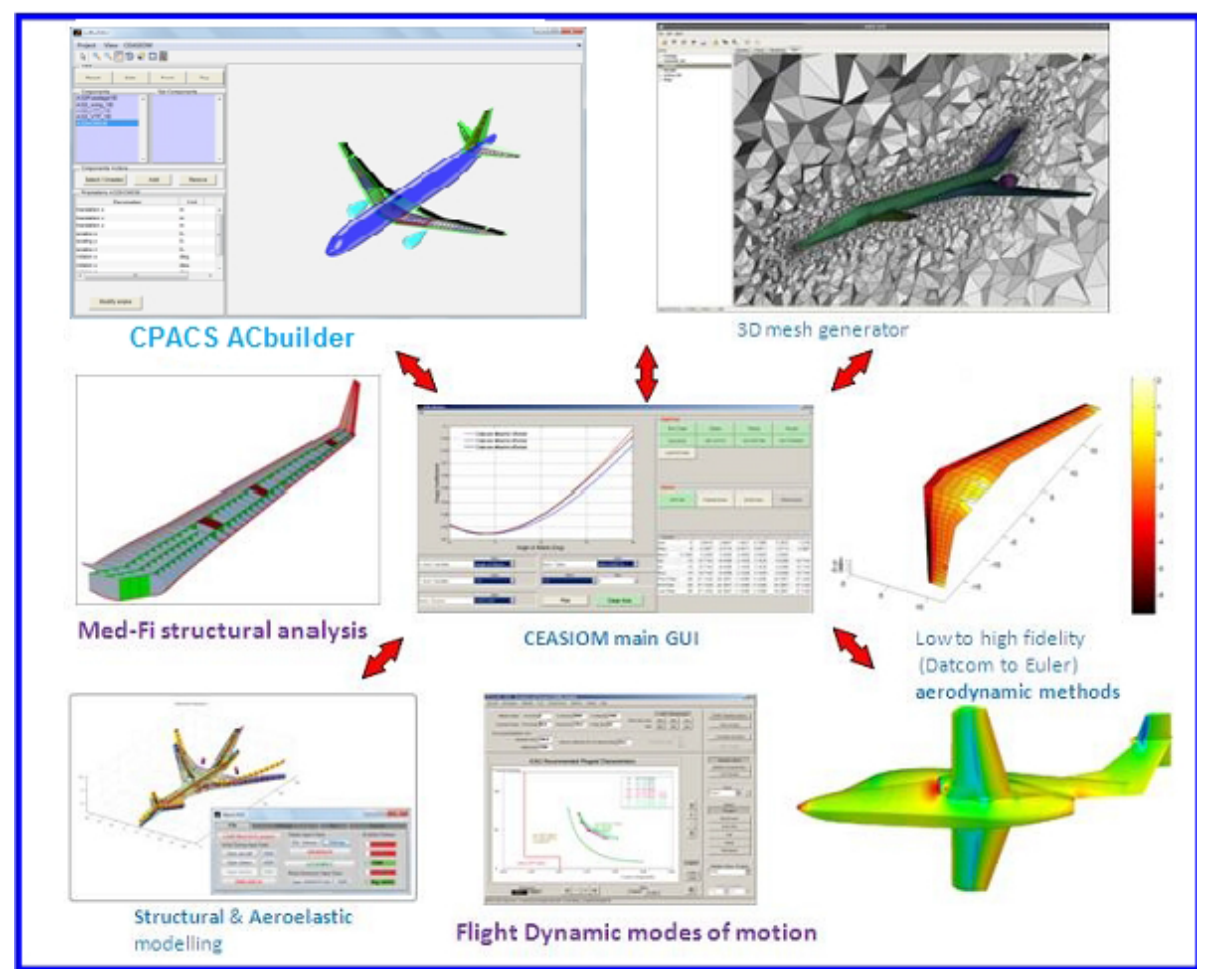

Figure 4. Core modules ACbuilder-sumo, AMB-CFD, NeoCASS and S\&C (SDSA ) in the CEASIOM software

\section{- Digital DATCOM}

- TORNADO Steady/unsteady vortex-lattice code (VLM) for low-speed (linear) aerodynamics and aeroelasticity

- Euler solver (EDGE CFD code in Euler mode) for inviscid flow cases where where total pressure and vorticity fields are too complex to model with isentropic equations e.g. at high speed or swirling flow. Examples of these are shock waves and propeller slipstreams

- RANS (Reynolds-Averaged Navier- Stokes) flow simulator (e.g. EDGE CFD code) for high fidelity viscous flow analysis at extreme flight conditions

- Stability and Control module S\&C (e.g. SDSA $^{19}$ ) A simulation and dynamic stability and control analyzer and flying-quality assessor. Includes:

- Performance prediction

- Test flights by six Degrees of Freedom flight simulation Stability Augmentation System (SAS)

- Aero-elastic module NeoCASS ${ }^{20}$ Quasi-analytical structural analysis methods that support aero-elastic problem formulation and solution.

CEASIOM is intended to support engineers in the conceptual/preliminary design process of the aircraft, with emphasis on the improved prediction of stability and control properties of elastic aircraft achieved by higher-fidelity methods than found in contemporary aircraft design tools. Moreover CEASIOM integrates into one application the main design disciplines, aerodynamics, structures, and flight dynamics, impacting on the aircraft's performance. It is thus a tri-disciplinary analysis toolbox brought to bear on the design of the aero-servo-elastic aircraft. ${ }^{22,28}$ CEASIOM however does not carry out the initial sizing of a baseline configuration, and thus needs to collaborate with a tool like ADAS, as was explained in Section I. 


\section{ADAS Design of a FAR 23 aircraft with 16 seats}

This section presents the design requirements for a FAR-23 compliant propeller driven aircraft with 16 seats. It explains the design procedure developed with ADAS, together with some results obtained, concerning the aerodynamic derivatives essential to the design at this stage. The procedure roughly follows the one indicated in Fig. 1, namely the two loops: pre-design and concept-design.

\section{A. Pre-design with ADAS}

\section{Initial weight estimation}

The design requirements for the selected case study are summarized in Table 1. The same table reports the assumptions used for the initial weight estimation. The weight estimation results are obtained with the well-known fuel fraction method ${ }^{30}$ and are shown in Figure 5. The selected airplane has a maximum take-off weight $W_{\mathrm{TO}}=5113 \mathrm{~kg}(11272 \mathrm{lb})$ and an operating empty weight $W_{\mathrm{OE}}=3225 \mathrm{~kg}(7110 \mathrm{lb})$.

Table 1. Design requirements and initial assumptions for weight estimation

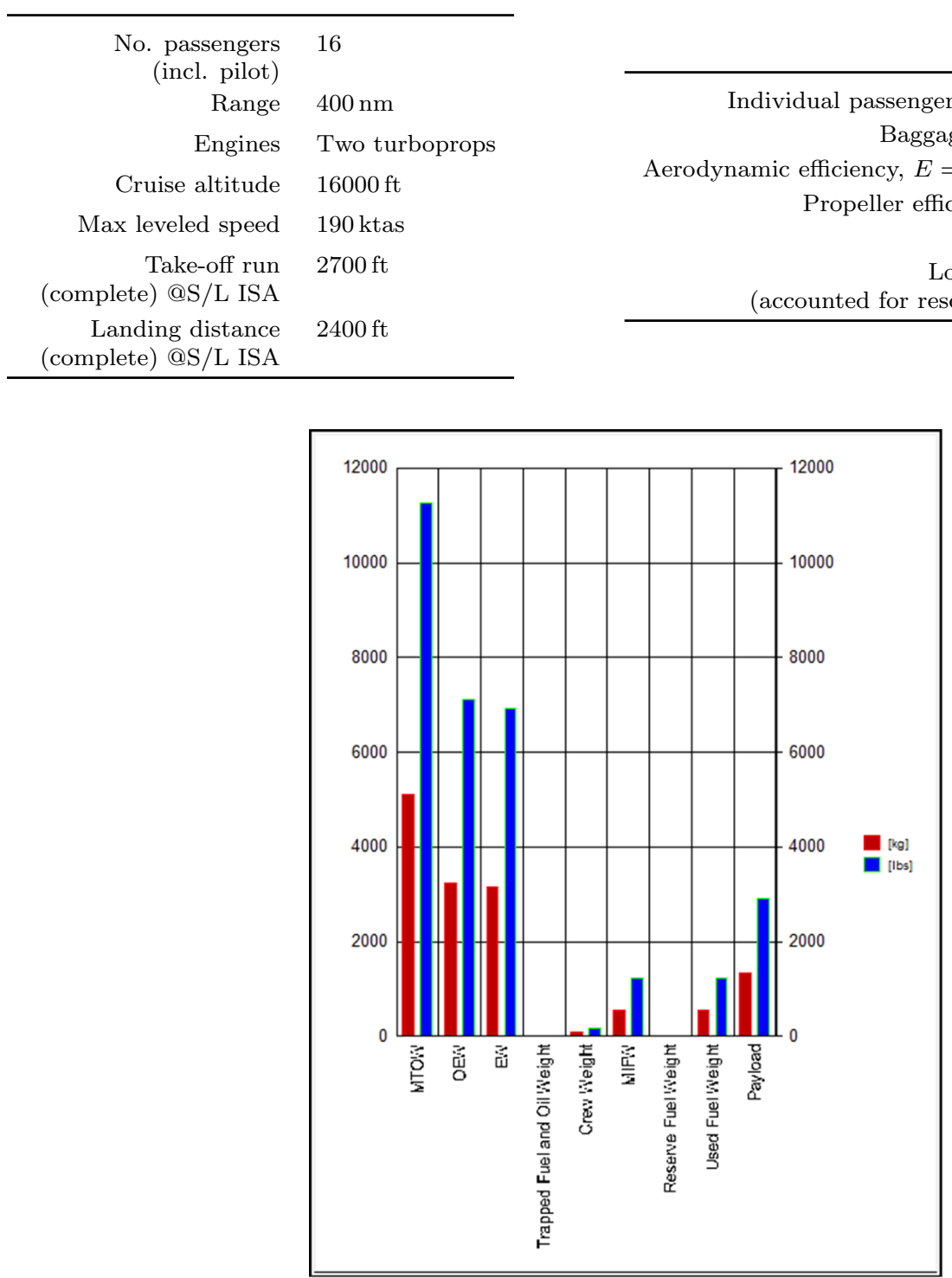

Figure 5. Aircraft preliminary weight estimation results. 


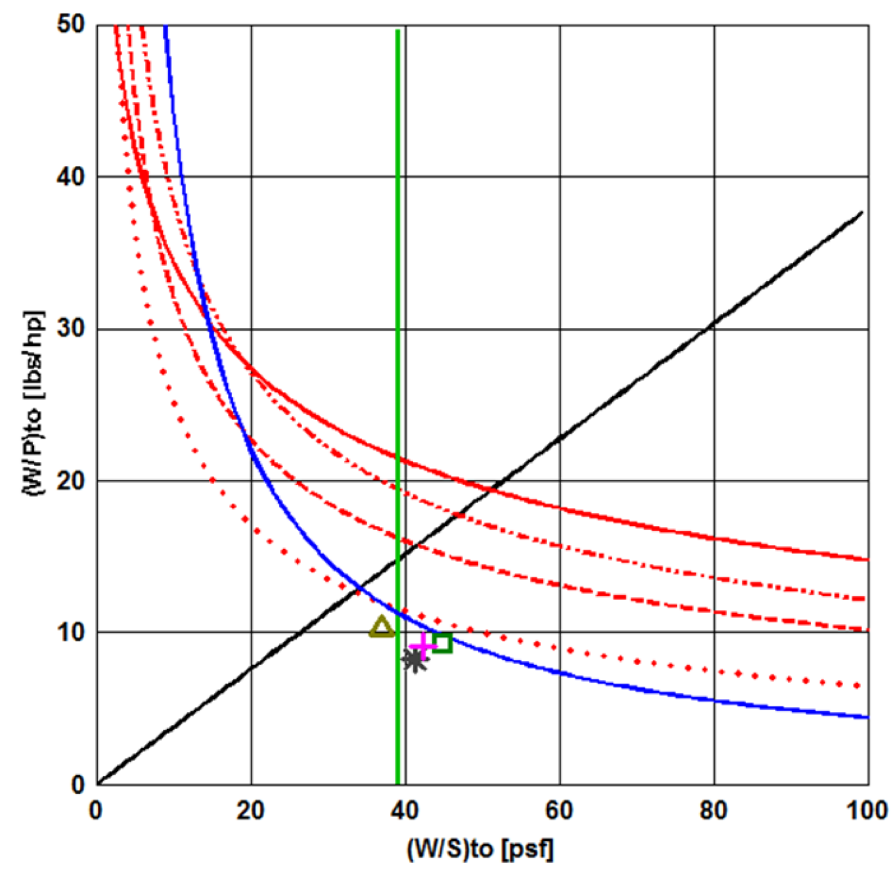

- Stall Speed (clean)

- -- Stall Speed (landing)

- TO 1 (2700 ft) CLM=1.90

- -- TO 2 (1600 ft) CLM=0.00

.... TO 3 (0 ft) $\mathrm{CLM}=\mathbf{0 . 0 0}$

— LDG 1 (2400 ft) CLM=2.35

- -- LDG 2 (0 ft) CLM $=0.00$

..... LDG 3 (0 ft) CLM $=0.00$

- FAR23.65 (1)

- -- FAR23.65 (2)

- . FAR23.67

-..- FAR23.77

Climb rate or Ceiling

- -- Climb gradient

... Time to climb

- $C R(190) h=16000 \% \mathrm{P}=100$

+ Embraer EMB121

** Piper PA-42 Cheyenne

-7- Fairchild Merlin

- Actual project

Figure 6. The typical carpet plot with the design point for the selected aircraft compared with similar airplanes.

\section{Wing and engine general sizing}

Considering take-off requirements and landing requirements, assuming a maximum lift coefficient of 1.90 at take-off and 2.35 at landing (full flap), given the FAR 23 climb limitations and the cruise condition as specified in the design requirements (Table 1), the parassite drag coefficient $C_{D 0}$ can been calculated.

The value $W_{\mathrm{TO}}=11272 \mathrm{lb}$ from design requirements, a statistical value of the wetted area $S_{\mathrm{wet}}=1393 \mathrm{ft}^{2}$, an assumed equivalent skin friction coefficient $C_{f e}=0.0052$ allow the calculation of an equivalent flat plate area $F=7.21 \mathrm{ft}^{2}$. For an assumed average wing loading $W / S=40 \mathrm{lb} / \mathrm{ft}^{2}$ the final result is a $C_{D 0}=0.0256$.

The value of $C_{D 0}$ is obtained for a wing aspect ratio $R=7.8$ and an Oswald factor of $e=0.80$, and assuming standard values of drag increment due to flap and landing gear to estimate the limitations due to FAR 23 climb requirements in all phases.

The analysis of all conditions with ADAS leads to the well-known carpet plot of Figure 6 . In the same plot are reported other design points representing similar airplanes. The aircraft under design should have the following characteristics:

$$
\left(\frac{W}{S}\right)_{\text {TО }}=37 \mathrm{lb} / \mathrm{ft}^{2} \quad\left(\frac{P}{W}\right)_{\text {TО }}=10.25 \mathrm{lb} / \mathrm{hp}
$$

From the value of the estimated weight the above values suggest a wing area $S=304.6 \mathrm{ft}^{2}=28.3 \mathrm{~m}^{2}$ and a necessary installed power $P=1100 \mathrm{hp}$ (two turboprop engines of $550 \mathrm{hp}$ each). The engines that could cope with the required power are for example the Pratt and Withney PT6A-20, as mounted in Beechcraft model 99 aircraft. The above values are summarized in Table 2.

Table 2. Wing and engine general sizing results.

\begin{tabular}{rl}
$(W / S)_{\mathrm{TO}}$ & $37 \mathrm{lb} / \mathrm{ft}^{2}$ \\
$(P / W)_{\mathrm{TO}}$ & $10.25 \mathrm{lb} / \mathrm{hp}$ \\
Wing area, $S$ & $304.6 \mathrm{ft}^{2}=28.3 \mathrm{~m}^{2}$ \\
Necessary installed power, $P$ & $2 \times 550 \mathrm{hp}$ \\
\hline
\end{tabular}




\section{B. Concept design with ADAS}

With the derived values of wing area $S$ and aspect ratio $A R$, assuming a standard value of taper ratio and a zero wing sweep $\Lambda_{c / 4}$ (this aircraft does not require sweep), the wing planform can be chosen and ADAS allows the calculations of all inherent aerodynamic characteristics. It is helpful to the designer to break down these characteristics into their constituent parts contributed by each of the main aircraft components: main wing, fuselage, nacelle and horizontal tail plane.

\section{Wing aerodynamic design}

The selected planform, shown in Figure 7, has an inner panel of constant chord and an outer tapered panel. Regarding wing sections, a NACA 23015 airfoil has been chosen at wing root and at wing kink, and a NACA 23012 has been selected at wing tip. The main wing dimensions are summarized in Table 3.

Table 3. Wing main dimensions

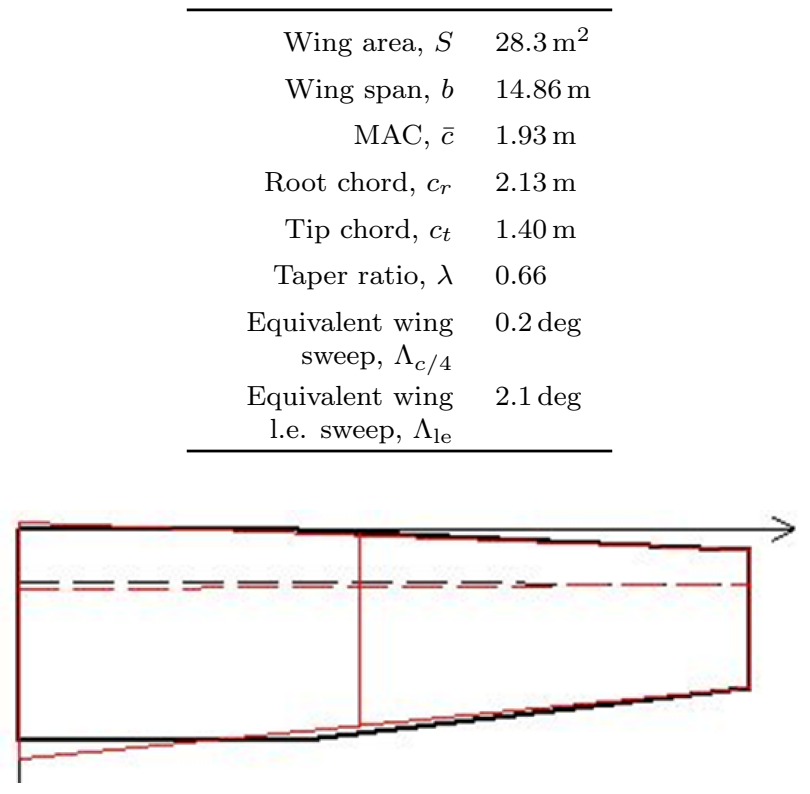

Figure 7. Wing planform drawing generated with ADAS.

The distribution of wing basic section lift coefficient, and the additional and total section lift coefficient distributions at different angles of attack are plotted in Figure 8. The analysis of wing span loading indicates the need of $2 \mathrm{deg}$ of wing twist (wash out). For a flight Mach number of 0.30 the wing aerodynamic characteristics are reported in Table 4. It has to be observed that NACA 230XX airfoil series have very limited zero-lift pitching moment coefficients. Figure 9 shows the wing lift curve and the wing pitching moment curve about the MAC quarter point.

\section{Fuselage sizing}

In ADAS the fuselage design is based on the assumed number of passengers, on the assumed seat pitch, and on standard space data for cabin arrangement. Assuming standard values of fineness ratio of fuselage nose and cone (1.4 and 2.0), a standard generic shape of the entire fuselage can be derived. The resulting characteristics for the aircraft under study are reported in Figure 10.

The aerodynamic analysis of the fuselage is performed with the classical Multhopp strip integration method (implementation of Munk formula) and leads to the results of Figure 11. The $C_{m}$ of the fuselage at $\alpha=0$ can be estimated in ADAS using the trip integration or the method suggested in Etkin's book. ${ }^{43}$ The figure shows the fuselage strip subdivision, the forward shift of wing-body aerodynamic center with respect to the isolated wing aerodynamic center, and the fuselage longitudinal and directional instability $\left(C_{m_{\alpha}}\right)_{\text {fus }}$ and $\left(C_{n_{\beta}}\right)_{\text {fus }}$. 


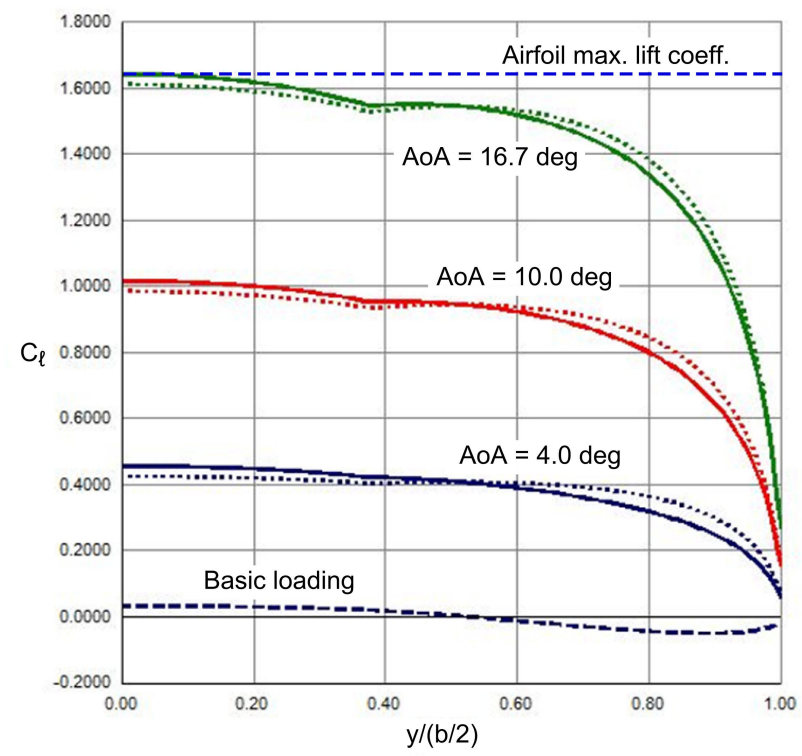

Figure 8. Wing spanwise loading and stall path.

Table 4. Wing aerodynamic characteristics

\begin{aligned} & \hline $\begin{array}{l}\text { Lift gradient, } C_{L_{\alpha}} \\ \text { (no fuselage effect) }\end{array} 0.084 \mathrm{deg}^{-1} \\ &$ Lift gradient, $C_{L_{\alpha}} 0.088 \mathrm{deg}^{-1} \\ &$ (with fuselage effect) \\ & Zero-lift AoA, $\alpha_{0 L}-0.5 \mathrm{deg} \\ &\left(\begin{array}{c}\text { w.r.t. root chord) } \\ \text { Wing-body max lift coeff. } \\ \left(C_{L \max }\right)_{\mathrm{WB}}\end{array}\right. 1.48 \\ &$ Wing aerod. center $0.244 \bar{c} \\ &$ position $\left(x_{\mathrm{ac}}\right)_{\mathrm{W}} \\ &$ Wing pitching moment -0.01 \\ & about aerod. center, $\left(C_{m_{\mathrm{ac}}}\right)_{\mathrm{W}} \\ &$\hline\end{aligned}

The fuselage pitching moment coefficient and drag coefficient curves versus body angle of attack are shown in Figure 12. The fuselage drag contribution to parasite drag coefficient $C_{D 0}$ can be considered about 0.0076 (76 drag counts) including upsweep and base drag.

\section{High-lift system and aileron design}

Wing flaps have been designed to achieve the requirements in term of maximum lift coefficient in landing conditions. A single slotted flap type has been chosen, with a $30 \%$ chord fraction. The flap is composed of two parts (inner and outer flap) with an overall spanwise extension that goes from $13 \%$ to $72 \%$ of the wing semispan. Each aileron has a 30\% chord fraction and extends from $72 \%$ up to $98 \%$ of the wing semispan. The high-lift system and aileron are represented in Figure 13.

The estimated gain in lift coefficient and the lift curve with flap extended in take-off $\left(\delta_{f}=15 \mathrm{deg}\right)$ and landing $\left(\delta_{f}=35 \mathrm{deg}\right)$ conditions are observed in Figure 14. The maximum lift coefficient with full flap is $C_{L \text { max,Land }}=2.30$.

The aileron control derivative has been calculated by considering also the reduction of control efficiency at higher deflection angles. The graph in Figure 15 shows a good value of the quantity $p b /(2 \mathrm{~V})$ at high aileron deflections. A maximum rolling speed of about $40 \mathrm{deg} / \mathrm{s}$ can be achieved with an aileron deflection of $18 \mathrm{deg}$ at a flight speed of $250 \mathrm{~km} / \mathrm{h}$ (close to maneuver speed $V_{A}$ ). Table 5 summarizes the calculation results related to the aileron analysis. 

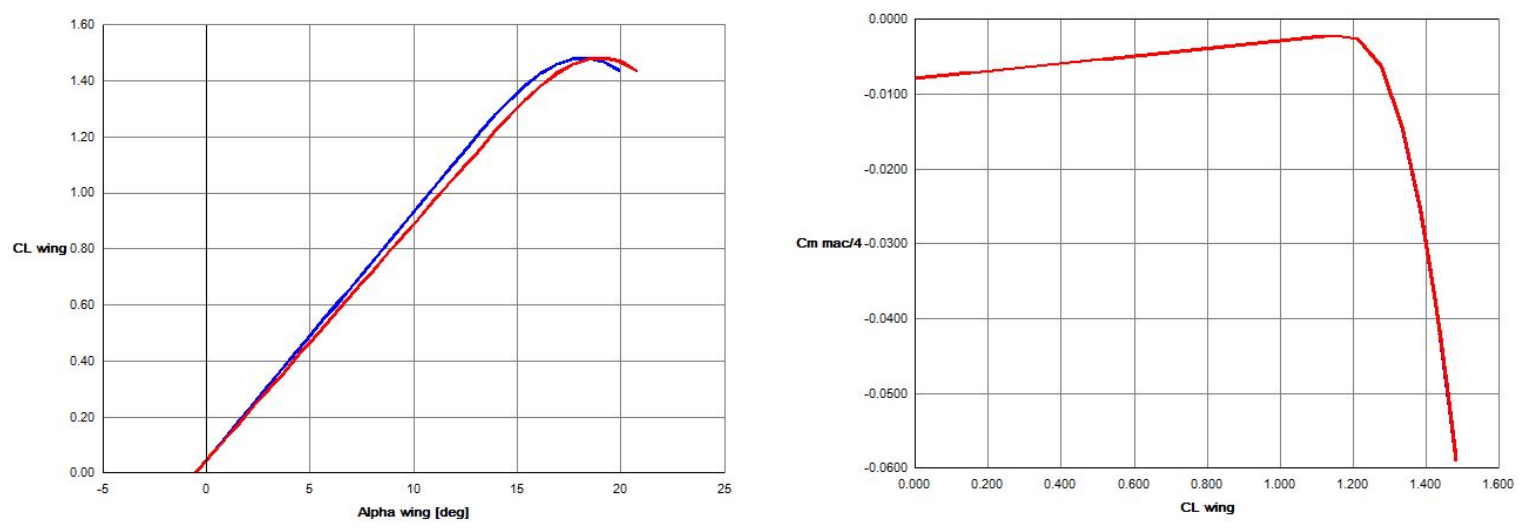

Figure 9. Wing lift and pitching moment curves.

Table 5. Aileron characteristics

\begin{tabular}{rl}
\hline Roll control power, $C_{l \delta_{a}}$ & $0.0027 \mathrm{deg}^{-1}\left(@ \delta_{a}=10 \mathrm{deg}\right)$ \\
Roll control power, $C_{l \delta_{a}}$ & $0.0018 \mathrm{deg}^{-1}\left(@ \delta_{a \max }=22 \mathrm{deg}\right)$ \\
Roll damping, $C_{l p}$ & -0.524 \\
$p b /(2 V)$ & $0.077\left(@ \delta_{a \max }=22 \mathrm{deg}\right)$ \\
\hline
\end{tabular}

\section{Horizontal tailplane design}

The input conditions for horizontal tail design are the following:

- $\quad$ wing-body-nacelle aerodynamic center position $x_{\mathrm{ac}, \mathrm{WBNac}}=0.097 \bar{c}$ (the fuselage leads to a forward shift of $13 \%$ and the two nacelles to about $2 \%$ of $\bar{c}$ ),

- $\quad$ zero-lift pitching moment coefficient $\left(C_{m \text { ac }}\right)_{\mathrm{WBNac}}=-0.0314$ (of which -0.0162 is due to the fuselage),

- $\quad$ horizontal tail sweep $\Lambda_{c / 4, \mathrm{H}}=12.5 \mathrm{deg}$,

- $\quad$ horizontal aspect ratio $R_{\mathrm{H}}=5.2$,

- $\quad$ elevator fraction chord equal to $30 \%$,

- $\quad$ maximum pitch up elevator deflection for equilibrium in landing conditions $\delta_{e \min }=-25$ deg.

According to the above assumptions, three design conditions have been considered:

1. minimum margin of static longitudinal stability, with stick free, equal to $5 \%$ (MSS $=-0.05$ ),

2. attainable equilibrium in landing condition at flight speed $V=1.1 V_{\mathrm{s}, \text { Land }}$, in ground effect,

3. take-off rotation at $V=1.05 V_{\mathrm{s}, \mathrm{TO}}$.

For the first condition the hinge moment derivatives have been estimated, considering standard values for the overhang: $c_{b} / c_{e}=0.19$ and Balance Ratio $=0.22$. These assumption have resulted in estimated values of $C_{h \alpha}=-0.225 \mathrm{rad}^{-1}$ and $C_{h \delta_{e}}=-0.409 \mathrm{rad}^{-1}$. Regarding the second condition, an increment of wing pitching moment due to full flap of $\left(\Delta C_{m}\right)_{f}=-0.25$ has been considered. For the third condition (rotation), a main landing gear placed at $40 \%$ of the chord has been assumed.

The first condition can be described by the following equation:

$$
\frac{x_{\mathrm{cg}}}{\bar{c}}=\frac{x_{\mathrm{ac}, \mathrm{WBNac}}}{\bar{c}}+\underbrace{\mathrm{MSS}}_{<0}+\eta_{\mathrm{H}} \frac{a_{\mathrm{H}}}{a}\left(1-\frac{\mathrm{d} \epsilon}{\mathrm{d} \alpha}\right)\left(1-\tau_{e} \frac{C_{h \alpha}}{C_{h \delta_{e}}}\right) \frac{l_{\mathrm{H}}}{\bar{c}} \frac{S_{\mathrm{H}}}{S}
$$




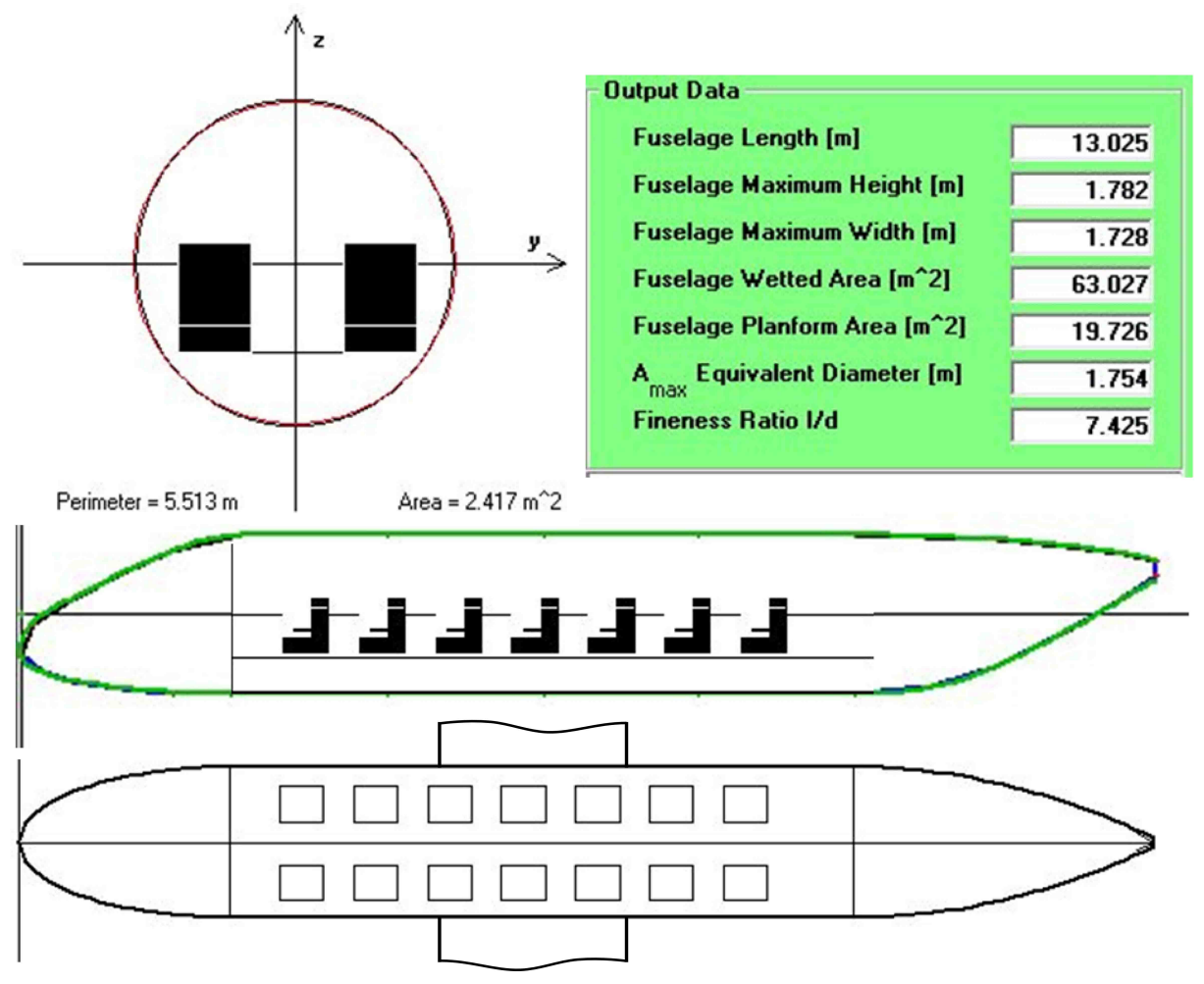

Figure 10. Fuselage views, seats and main dimensions in ADAS.

The second condition (equilibrium in landing) is expressed by the following equation:

$$
\begin{aligned}
& C_{m \mathrm{cg}}=\left(C_{m \mathrm{ac}}\right)_{\mathrm{WBNac}}^{\mathrm{flap}}+\left[\frac{\left.x_{\mathrm{cg}}-x_{\mathrm{ac}, \mathrm{WBNac}}^{\mathrm{flap}}-\eta_{\mathrm{H}} \frac{a_{\mathrm{H}}}{a}\left(1-\frac{\mathrm{d} \epsilon}{\mathrm{d} \alpha}\right)_{\text {g.e. }} \frac{l_{\mathrm{H}}}{\bar{c}} \frac{S_{\mathrm{H}}}{S}\right] C_{L \text { eq }}}{}\right. \\
&-K \eta_{\mathrm{H}} a_{\mathrm{H}} \frac{l_{\mathrm{H}}}{\bar{c}} \frac{S_{\mathrm{H}}}{S}\left(i_{\mathrm{H} 0}+\tau_{e} \delta_{e}-\left(\alpha_{0 L}\right)_{\mathrm{W}}^{\mathrm{flap}}\right)=0
\end{aligned}
$$

The downwash gradient has to be estimated also in ground effect and the following additional data have been considered: $(\mathrm{d} \epsilon / \mathrm{d} \alpha)_{\text {Cruise }}=0.343$ (first cond.), $(\mathrm{d} \epsilon / \mathrm{d} \alpha)_{\text {Land }}=0.305$, tailplane incidence $i_{\mathrm{H} 0}=-2 \mathrm{deg}$, wing zero-lift body angle of attack with full flap $\left(\alpha_{0 L}\right)_{\mathrm{W}}^{\text {flap }}=-10 \mathrm{deg}$, wing incidence $i_{\mathrm{W}}=2 \mathrm{deg}$.

The above three design conditions, when equations (1)-(2) are applied, lead to the graph of Figure 16(a). The plot shows the selected horizontal tail area to wing area ratio $S_{\mathrm{H}} / S=0.24$, which leads to a good excursion of the center of gravity.

The final designed value are: a tail horizontal surface $S_{\mathrm{H}}=6.79 \mathrm{~m}^{2}$ and a horizontal tail volumetric coefficient $\mathcal{V}_{\mathrm{H}}=0.89$, with a resulting neutral point stick fixed at $47 \%$ of the MAC. A sketch of the tailplane with the main design results is shown in Figure 16(b).

For a center of gravity placed at $25 \%$ of MAC the graphs of Figure 17 show a break down of contributions to aircraft pitching moment coefficient. Table 6 summarizes the airplane longitudinal characteristics.

Calculations performed at different elevator deflections, also including nonlinear effect at higher values of $\delta_{e}$, lead to the graphs of Figure 18. Two conditions are shown, i. e. the cruise condition with a Mach number of 0.30 and a center of gravity at $25 \%$ of MAC, and a landing condition with a Mach number of 0.15 , a center of gravity at $18 \%$ of MAC and flap fully extended. These curves show that the trimmed lift coefficient of 2.3 can be achieved with a $\delta_{e}=-25 \mathrm{deg}$. The design elevator control power in the linear range is $C_{m \delta_{e}}=-0.035 \mathrm{deg}^{-1}$. 

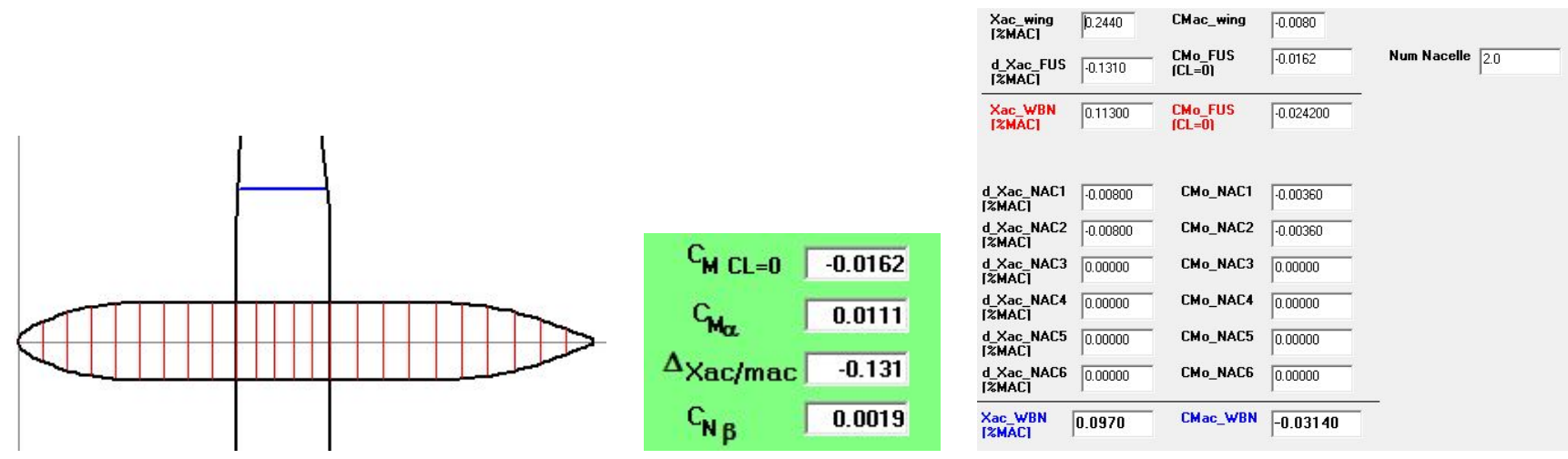

Figure 11. Fuselage subdivision in strips for Multhopp integration method and Etkin's method ${ }^{43}$
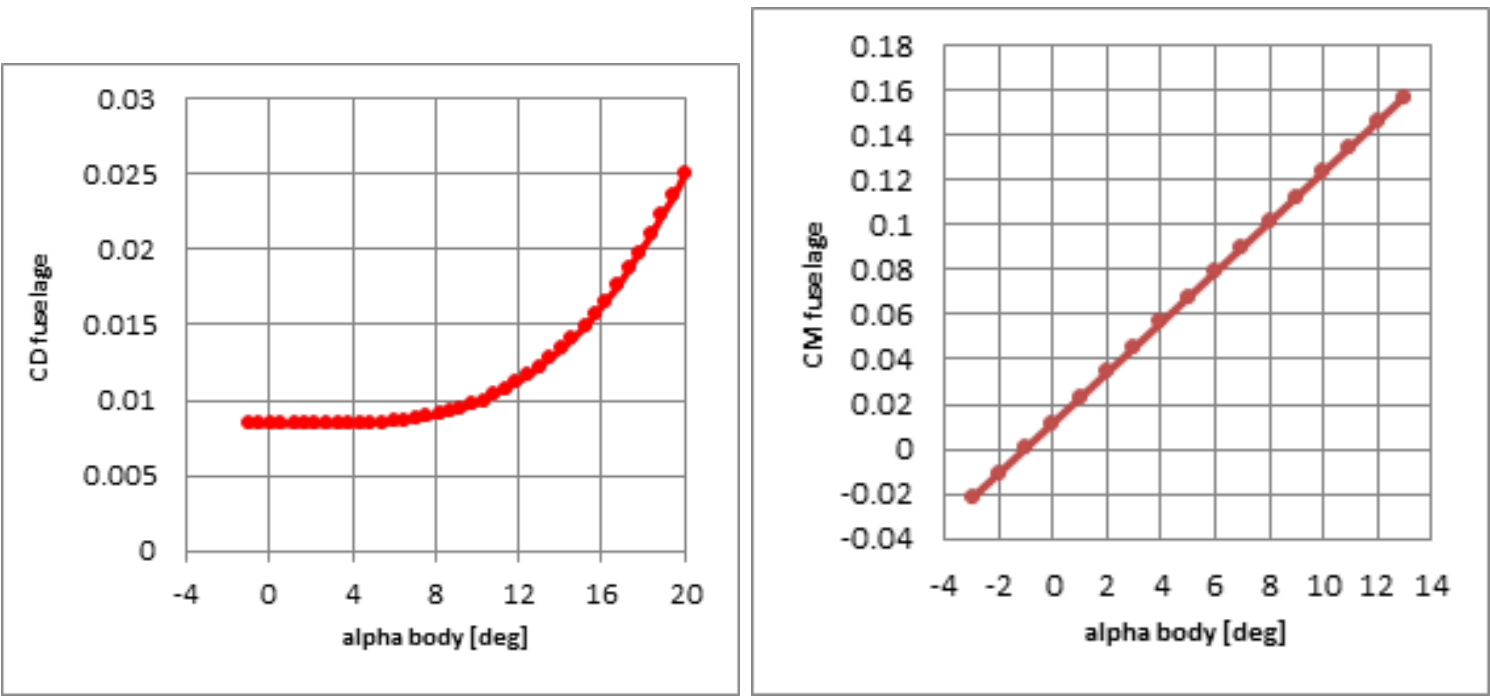

Figure 12. Fuselage drag coefficient and pitching moment coefficient.

\section{Vertical tailplane design}

The vertical tailplane has been designed to accomplish the minimum control speed ( $V_{\mathrm{mc}}$, one engine out condition in take-off) and to control the landing with a severe sideslip condition.

To this purpose the curves of yawing moment coefficient (aerodynamic and due to thrust versus speed) of Figure 19 have been constructed. The equilibrium at $V_{\mathrm{mc}}$, assuming $V_{\mathrm{mc}}=1.14 V_{\mathrm{s}, \mathrm{TO}}$, is obtained with $S_{\mathrm{V}}=5.60 \mathrm{~m}^{2}$. For higher confidence and to improve control in landing (ratio between $C_{n \beta}$ and $C_{n \delta_{r}}$ ), a higher vertical tail area $S_{\mathrm{V}}=5.80 \mathrm{~m}^{2}$ has been assumed as design condition. The selected tail gives a ratio $S_{\mathrm{V}} / S=0.205$ and a volumetric coefficient $\mathcal{V}_{\mathrm{V}}=0.088$

For a linear aerodynamic behavior, i. e. for sideslip angles up to $\beta=10 \mathrm{deg}$ and rudder deflections $\delta_{r}=10 \mathrm{deg}$, and at cruise Mach number the main aerodynamic characteristics of the vertical tail are summarized in Table 7 .

\section{Lateral stability (dihedral effect)}

From the above design choices ADAS estimates the following value of dihedral effect: $C_{l \beta}=-0.0020 \mathrm{deg}^{-1}$.

\section{Estimated drag polar}

Figure 20 summarizes the contributions to the total parasitic drag coefficient, which is ADAS gives equal to $C_{D 0}=0.0270$ coupled with an estimated Oswald factor $e=0.82$. 


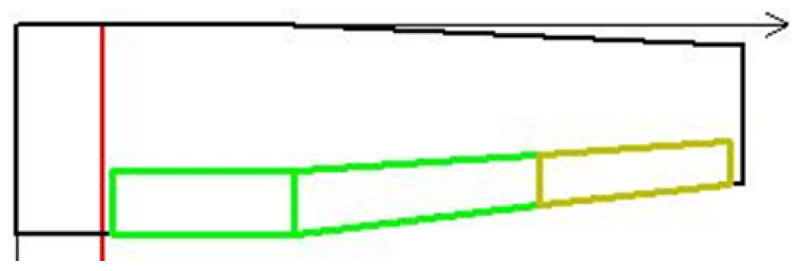

Figure 13. Wing high-lift and aileron designs.

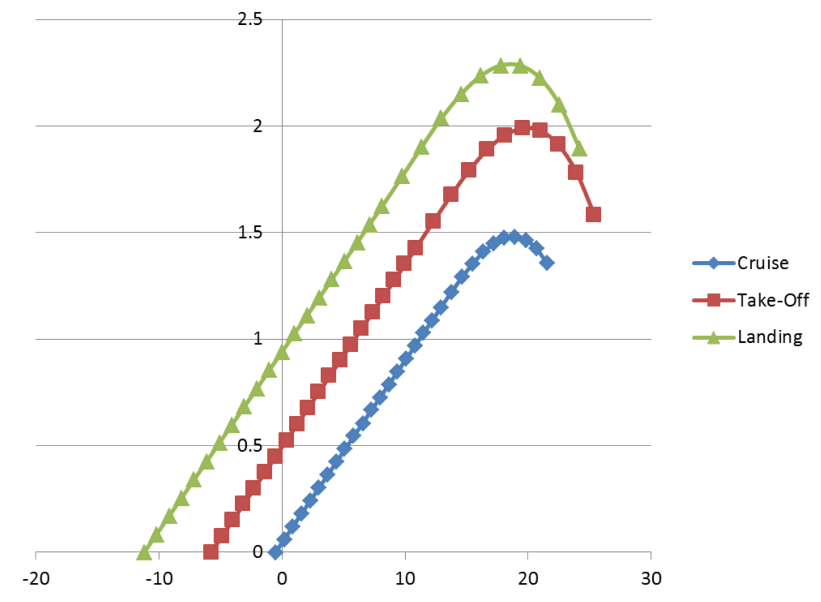

Figure 14. Wing lift curves for three flight conditions: cruise, take-off and landing.

\section{External aerodynamic shape: 3-view configuration}

The three-dimensional representation of the external shape of the aircraft is shown in Figure 21, a 3-view of the configuration along with a 3D orthographic view. Note however that the surfaces in this figure are not trimmed, they may overlap, and there may be holes in them. Thus more work must be done before this configuration can be gridded for CFD analysis.

\section{Down-select Configuration to CEASIOM}

Figure 21 shows the ADAS 3-view drawing of the final conceptual design for the twin-propeller aircraft TWINPROP-16. This is the configuration that is down-selected from conceptual design and is now ready for preliminary design, as illustrated in Fig. 1.

A primary goal of preliminary design is to obtain the final wing design with optimized performance: e.g. maximized $L / D)$, or minimized $C_{D}$, or an Oswald efficiency factor $e$ near 1 . The wing airfoils are

Table 6. Airplane longitudinal characteristics for a CG placed at $25 \%$ of MAC.

\begin{tabular}{rl}
\hline $\begin{array}{r}\text { Airplane long. static stability, } C_{m_{\alpha}} \\
\text { (in AoA linear range) }\end{array}$ & $-0.0261 \mathrm{deg}^{-1}$ \\
Body + Nacelle instability, $\left(\Delta C_{m_{\alpha}}\right)_{\mathrm{BNac}}$ & $+0.0130 \mathrm{deg}^{-1}$ \\
$\quad$ (in AoA linear range) & \\
Tailplane stability contrib., $\left(\Delta C_{m_{\alpha}}\right)_{\mathrm{H}}$ & $-0.0380 \mathrm{deg}^{-1}$ \\
$\quad$ (in AoA linear range) & \\
Airplane lift gradient, $C_{L_{\alpha}}$ & $0.102 \mathrm{deg}^{-1}$ \\
$\quad$ (incl. hor. tail) & \\
Neutral point stick fixed, $x_{N}$ & $0.51 \bar{c}$ \\
(in AoA linear range) & \\
\hline
\end{tabular}

13 of 26

American Institute of Aeronautics and Astronautics 

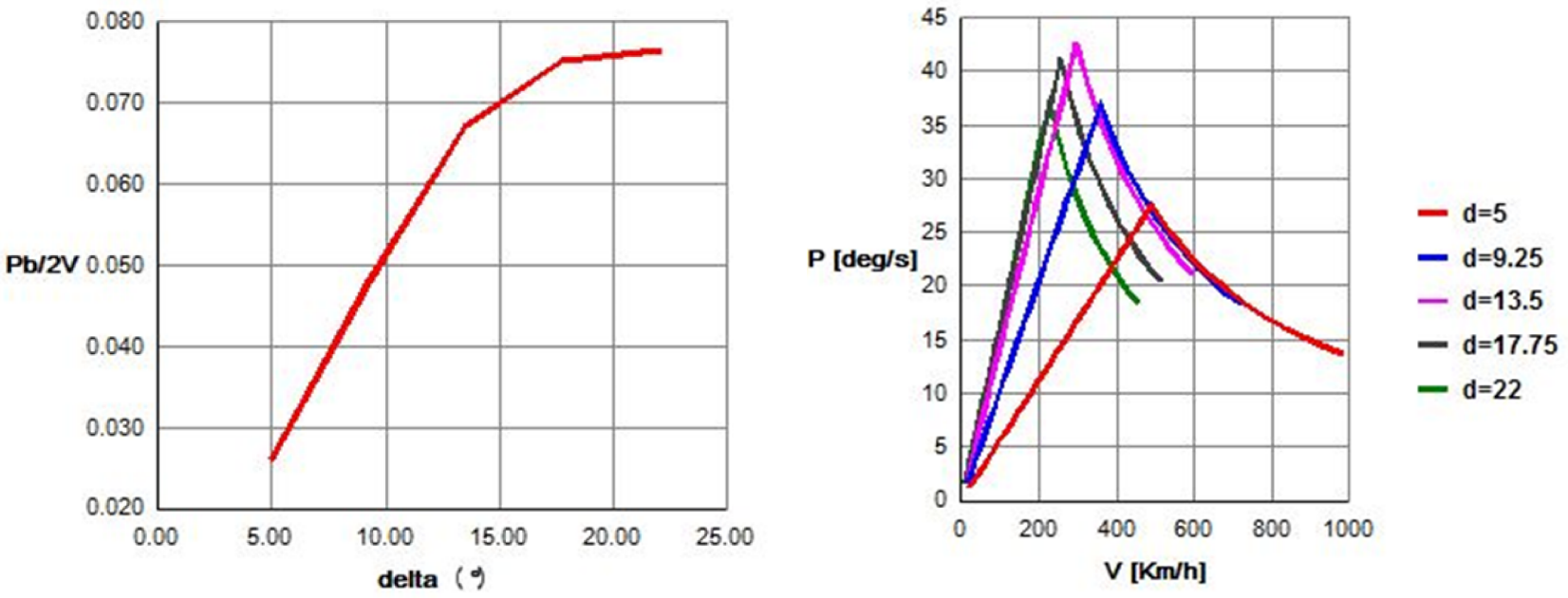

Figure 15. Aileron effectiveness analysis.

Table 7. Vertical tail design results

\begin{tabular}{rl}
\hline Yaw control power, $C_{n \delta_{r}}$ & $-0.0031 \mathrm{deg}^{-1}$ \\
Airplane directional stability, $C_{n \beta}$ & $0.0022 \mathrm{deg}^{-1}$ \\
Body + nacelle contrib., $\left(C_{n \beta}\right)_{\mathrm{BNac}}$ & $-0.0019 \mathrm{deg}^{-1}$ \\
Vertical tail contrib., $\left(C_{n \beta}\right)_{\mathrm{V}}$ & $0.0042 \mathrm{deg}^{-1}$ \\
\hline
\end{tabular}

chosen by a skillful engineer as the initial design, which may not be optimum, but can be used as a good starting point. Take the TWINPROP-16 aircraft for example, the airfoils of the wing are typical NACA 23015 and 23012, with wing incidence $i_{\mathrm{W}}=2^{\circ}$. Then it should be put in an optimization loop to determine the optimized airfoil shapes (thickness and cambers), twist distributions according to the limits of $C_{L}, C_{m}$, bending moment, span loading, fuel tank volumes etc. for corresponding flight conditions. Usually to get a well designed wing a multi-level optimization ${ }^{42}$ should be considered, namely, optimizations for cruise, takeoff and landing. For low speed aircraft a good number to assess the design is the Oswald factor $e$, which is not so high $(e=0.82)$ for the ADAS configuration. There exist some design codes that give much better results using simple lifting line theory by defining an elliptical wing span loading which results a minimum induced drag. Nangia ${ }^{41}$ and Lamer ${ }^{40}$ are two good examples.

Another goal is to determine a database of aerodynamic forces and moments that cover sufficiently the flight envelope so that it is appropriate input to a flight simulator for the study of the vehicle's performance and handling qualities. Tables 8 indicate how the aerodynamic database computed in CEASIOM is organized: Table 8.I shows the static and quasi-static stability coefficients, Table 8.II shows the control coefficients, and Table 8.III shows the dynamic stability derivatives where $\alpha$ is the angle of attack, $M$ is the Mach number and $\beta$ the side slip angle, $q, p$ and $r$ are the three rotations in pitch, roll and yaw. The three control surfaces that can be deflected are the elevator $\left(\delta_{e}\right)$, the rudder $\left(\delta_{r}\right)$ and the aileron $\left(\delta_{a}\right)$. The coefficients must be obtained for each of these three parameters throughout the flight envelope, hence the data is voluminous.

Yet a third goal is to determine the aerodynamic loads acting on the skin so that an appropriate structure can be devised to bear these loads. The simplest example of this is the span loading on the main wing and its associated root bending moment.

$\mathrm{SAAB}^{39}$ has found the propeller wash over the wing was important in design of SAAB2000, and this work demonstrated that the propeller can be modeled adequately in inviscid flow by Euler equations. The propeller disk for TWINPROP-16 is modeled and analyzed in CEASIOM.

The starting point for working towards all these goals begins with the external shape of the baseline configuration, and constructing a computation grid upon it for physics-based analysis. However, the 3-view configuration that is typically produced in conceptual design is not a geometry model of trimmed surfaces that is water-tight, i.e. a surface mesh cannot be generated upon the surfaces without additional work. This 


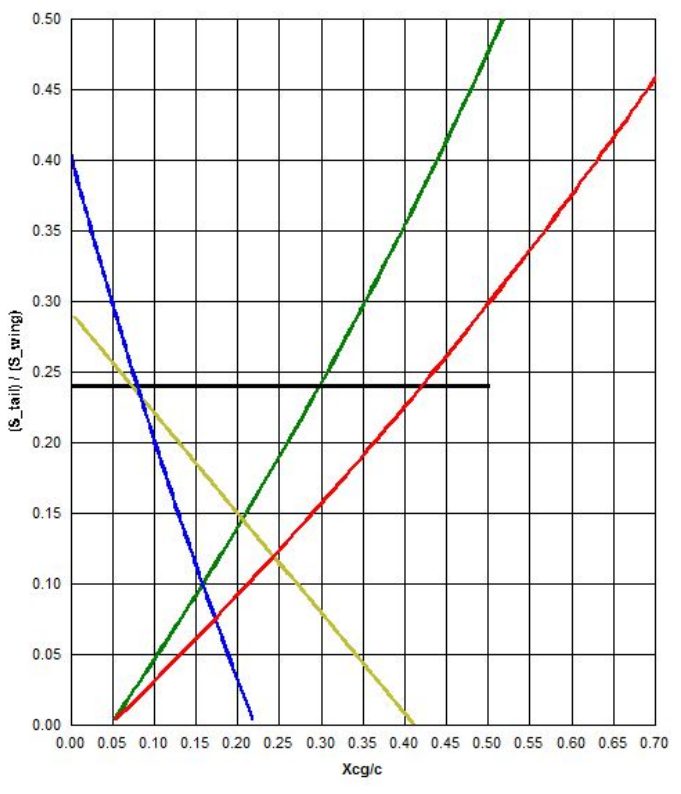

(a) Horizontal tail design

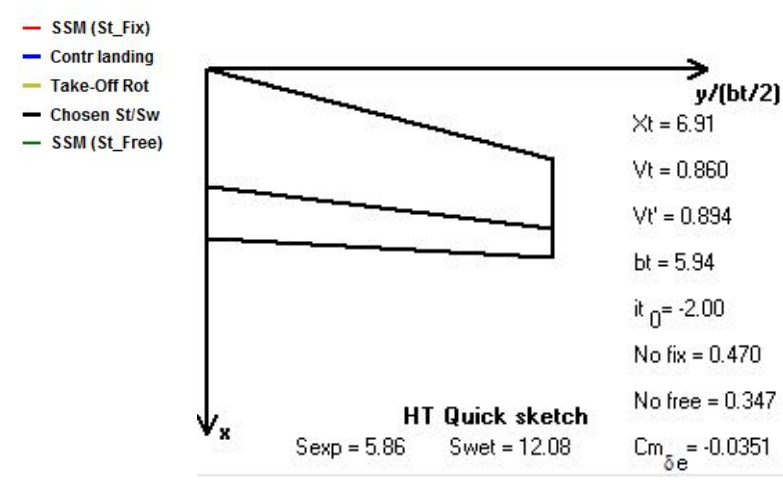

(b) Horizontal tail sketch

Figure 16. ADAS horizontal design and its sketch

\begin{tabular}{c|c|c|c|c|ccc|cccccc|}
\hline [I, Stability coefficients table ] & Alpha & Mach & Beta & $q$ & $p$ & $r$ & $C_{L}$ & $C_{D}$ & $C_{m}$ & $C_{Y}$ & $C_{\ell}$ & $C_{n}$ \\
\cline { 2 - 12 } & $\mathrm{x}$ & $\mathrm{x}$ & $\mathrm{x}$ & - & - & - & $\mathrm{x}$ & $\mathrm{x}$ & $\mathrm{x}$ & $\mathrm{x}$ & $\mathrm{x}$ & $\mathrm{x}$ \\
$\mathrm{x}$ & $\mathrm{x}$ & - & $\mathrm{x}$ & - & - & $\mathrm{x}$ & $\mathrm{x}$ & $\mathrm{x}$ & $\mathrm{x}$ & $\mathrm{x}$ & $\mathrm{x}$ \\
$\mathrm{x}$ & $\mathrm{x}$ & - & - & $\mathrm{x}$ & - & $\mathrm{x}$ & $\mathrm{x}$ & $\mathrm{x}$ & $\mathrm{x}$ & $\mathrm{x}$ & $\mathrm{x}$ \\
$\mathrm{x}$ & $\mathrm{x}$ & - & - & - & $\mathrm{x}$ & $\mathrm{x}$ & $\mathrm{x}$ & $\mathrm{x}$ & $\mathrm{x}$ & $\mathrm{x}$ & $\mathrm{x}$ \\
\hline
\end{tabular}

[III, Dynamic stability derivatives table ] \begin{tabular}{|c|ccccccc|}
\hline Mach & $C_{m_{\dot{\alpha}}}$ & $C_{Z_{\dot{\alpha}}}$ & $C_{X_{\dot{\alpha}}}$ & $C_{Y_{\dot{\beta}}}$ & $C_{\ell_{\dot{\beta}}}$ & $C_{n_{\dot{\beta}}}$ \\
\hline $\mathrm{x}$ & $\mathrm{x}$ & $\mathrm{x}$ & $\mathrm{x}$ & $\mathrm{x}$ & $\mathrm{x}$ & $\mathrm{x}$ \\
\hline
\end{tabular}

Table 8. Structure of the aerodynamic database constructed in CEASIOM for use in the flight simulation SDSA module.

is the first task in preliminary design, to prepare the geometry model for meshing. In order to do this, we first must import the 3 -view geometry into CEASIOM.

\section{A. Import ADAS Geometry into CEASIOM}

The gateway to physics-based analysis is a 'meshable' CAD model. The ADAS geometry is defined by components stored in .def files, which provide fundamental geometric information of the whole aircraft. The wings are defined by describing the planform with sectional chord lengths, leading and trailing edge positions, wing incidence and dihedral angles. The airfoil coordinates for each section are appended in the end of the file. The fuselage and the nacelles are defined in a different way, by specifying the coordinates of each section along the body-wise direction (x-axis). This is consistent with the corresponding geometry defined in sumo, which is the most difficult part to model by a mouse-driven process due to the complexity of the geometry. A (C)MATLAB script is dedicated to read and convert the def files to a watertight sumo aircraft geometry 


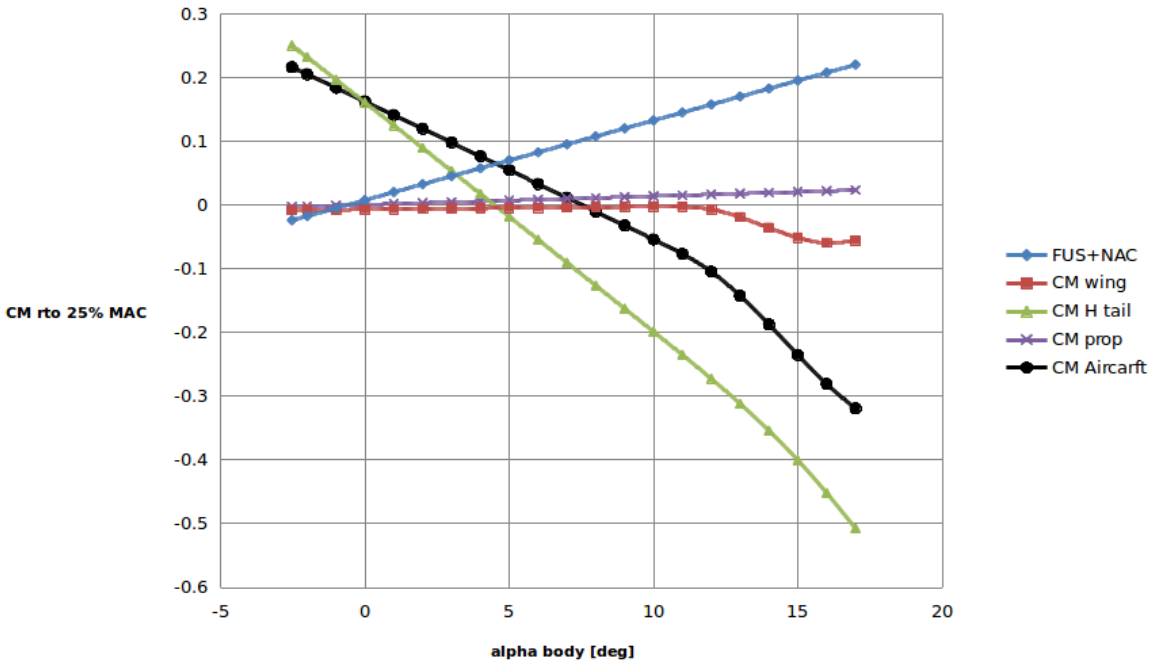

Figure 17. Break down of contributions to aircraft pitching moment coefficients for CG at $25 \%$ of MAC.

in an automatic manner.

Figure 22 shows the watertight NURBS model created in CEASIOM-sumo. This can be exported as an IGES file for further physics-based analysis.

This watertight model is 'meshable' by definition and it opens the door to the higher-fidelity Euler solver in CEASIOM once the volume mesh is generated automatically by sumo-tetgen. ${ }^{27}$ The spinners and the propellers are modified/created later by human interaction.

\section{B. Propeller disk model}

The way of modeling the propeller disk in this paper is focus on using CEASIOM-sumo, i.e., adding momentum by specifying the mass flow through the propellers. The thrust is calculated based on the estimation from ADAS to balance the drag while maintaining a steady and level flight.

Table 9. Data from ADAS for TWINPROP16 to calculate the thrust

\begin{tabular}{cccccc}
\hline$U$ & $m[\mathrm{~kg}]$ (cruise) & $C_{D 0}$ (clean cruise) & $S_{\text {ref }}\left[\mathrm{m}^{2}\right]$ & $e$ (Oswald factor) & $R$ \\
\hline 100 & 4500 & 0.03 & 28.29 & 0.8 & 7.806 \\
\hline
\end{tabular}

The $C_{L}$ for a steady and level flight is estimated by

$$
C_{L}=0.5 \frac{m \cdot g}{\rho U^{2} S_{r e f}}
$$

thus, the $C_{D}$ is estimated by lifting-line theory

$$
C_{D}=C_{D 0}+\frac{C_{L}^{2}}{e \cdot \pi \cdot \AA}
$$

The diameter of the propeller disk is assumed to be 1.58 meter, the mass flow $(\dot{m})$ can be calculated according to the thrust which should balance the total drag provided there is a steady and level flight. The total mass flow will be

$$
\dot{m}=\rho A u
$$

where $A$ is the disk area which is unchanged from inlet to outlet, and $u$ is the flow velocity (assuming x-axis). The mass flow $\dot{m}_{\text {inlet }}=\dot{m}_{\text {outlet }}$ due to the continuity equation and unchanged $A$ as well. The thrust $T$ is calculated by the change of momentum flux. 


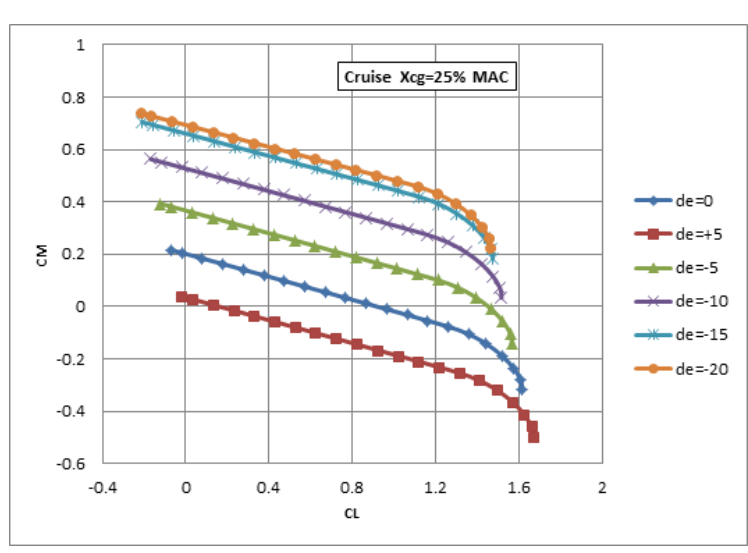

(a) $\mathrm{CG}$ at $25 \%$ of MAC, cruise condition, Mach $=0.30$

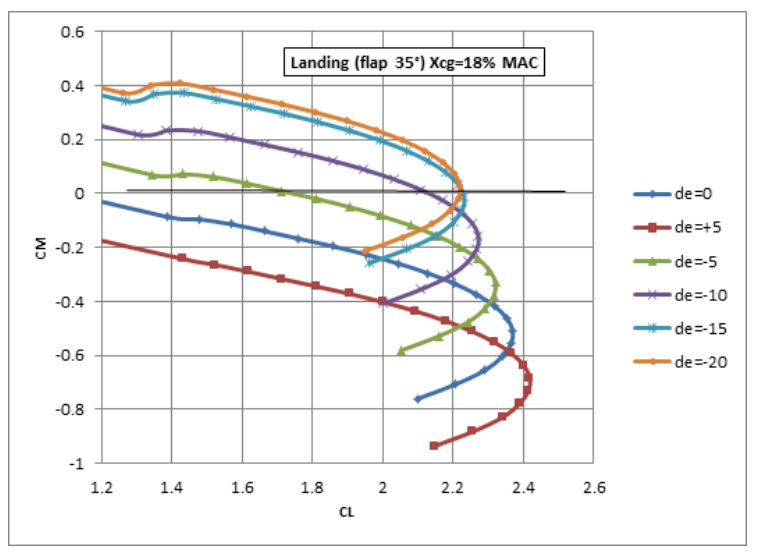

(b) CG at $18 \%$ of MAC (max fwd), landing condition, Mach $=0.15$, full flap

Figure 18. Pitching moment coefficient versus lift coefficient.

$$
T=\dot{m}\left(u_{2}-u_{1}\right)
$$

Table 10. Data from ADAS for TWPROP16 to calculate the thrust

\begin{tabular}{|c|c|c|c|}
\hline total drag $[\mathrm{kN}]$ & $T[\mathbf{k N}]$ (per prop) & $\dot{m}$ per prop & $\dot{m}$ OEI \\
\hline 5.7 & 2.85 & 260 & 283 \\
\hline
\end{tabular}

By defining the thrust coefficient $C_{F}$ as

$$
C_{F}=\frac{T}{0.5 \rho U^{2} A}
$$

The thrust coefficient for each propeller is $C_{F}=0.24$, if one engine fails, the thrust coefficient for the live engine will be dramatically doubled, $C_{F}=0.48$.

\section{Volume mesh around configuration with propeller disk}

First a mesh (around 5 million nodes) is generated automatically with the CEASIOM tool sumo, displayed in figure 23, with propellers modeled directly from sumo as finite thickness disks by specifying the mass flow in-and-out to model the pressure jump after the propellers. The propeller is simplified as an actuator disk, neglecting the rotation imparted to the flow. Since the Mach number is low, the flow is nearly incompressible, and the velocity is almost constant (mass conservation) if the propeller effects are not considered. However, the pressure jumps across the actuator disk to provide the desired thrust.

An alternative is to allow the usage of disk boundary condition in the Edge Euler in the CEASIOM. The propeller is represented by a two-sided disk between two cells in the grid modeling in ICEM-CFD with both sides are topologically the same, as Lötstedt did in his paper. ${ }^{39}$ This will be done in a future paper.

\section{Computed Euler solutions around TWINPROP-16}

Euler analysis was carried out at Mach number 0.303 for 16 different cases, shown in Table 11 for propeller both OFF and ON, as indicated. These 16 cases are a small first step towards constructing a full aerodynamic database, as discussed above.

Figure 24 shows the pressure distributions from Euler with propellers ON and OFF. For the propeller OFF case, the flow just goes through the propeller. There is a pressure jump across the propeller disk, which compresses the air density after the disk, in order to maintain the continuity equation, the velocity has to decrease, since $A$ is unchanged. The total energy is also increased with a step since the amount of power is put in the flow field by the actuator disk. 


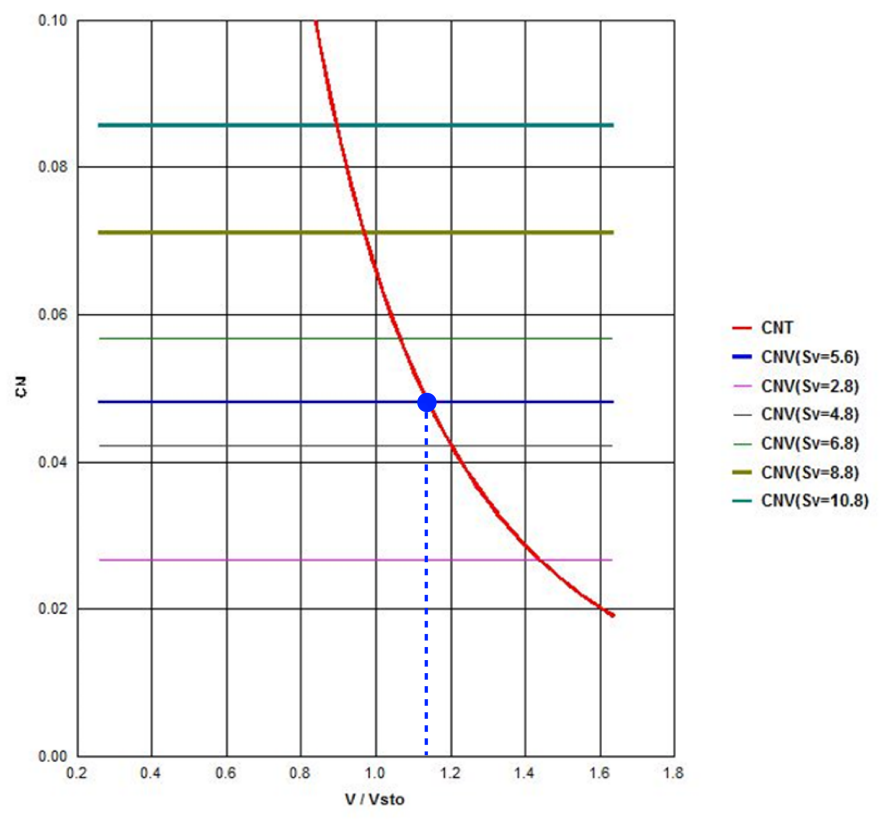

Figure 19. Vertical tail design.

From figure 25 we can see that the pressure decreases to the surrounding value far downstream, resulting a velocity increase due to momentum conservation. A time accurate equation should be solved if the propeller's swirl is under investigation. For low Mach number the flow generated by propeller is well described by the actuator disk theory as stated above.

Aerodynamic coefficients are computed in CEASIOM VLM-Tornado and Euler-Edge respectively, and both sets of results are comparable if the differences in modeling are taken into account. Therefore only Euler results will be shown here and discussed.

The deflected control surfaces are computed in Edge by transpiration boundary conditions, i.e. they are not physically deflected in the mesh. CEASIOM now has the functionality to physically deflect the control surfaces in the mesh, ${ }^{24}$ and this will be a topic for a future paper.

The propeller energizes the flow downstream, the accelerated flow on the upper surface of the wing which is resulted by a greater pressure difference on both surfaces generates more lift for the main wing, and also for the horizontal tail (figure 24). Figure 26 shows that the longitudinal forces and moments computed from both propeller ON and OFF configurations. Note that the transpiration boundary condition gives around $30 \%$ less elevator efficiency than ADAS semi-empirical estimations.

\section{Comparisons of ADAS and CEASIOM Results}

Table 11 lists the 16 cases run with the CEASIOM-Euler solver, which provides comparison of the aerodynamic coefficients and derivatives from ADAS and CEASIOM. The cases in the first three rows in Table 11 study static longitudinal stability, the next four cases study static lateral stability while the remaining 3 sets of 3 cases study pitch, yaw and roll control, respectively. Both propeller OFF and ON cases are run, with only elevator deflected for cases that propellers are ON. The coefficients and their derivatives from most of the cases agree well between ADAS and CEASIOM (Prop OFF) estimations, while for the Prop ON cases CEASIOM gives significant higher deriviatives than ADAS.

The matrix of aerodata is a very small subset, but could be a start, and it can provide the stability derivatives for linear aerodynamics, see table 12. To get a quick check, CEASIOM-TORNADO can used, which only accounts for the lifting surfaces (no thickness). In a future paper a full aerodata table will be made, which will be imported into SDSA for stability \& control analysis. At this stage some basic analysis still can be done, for example, the trimmed aircraft can be achieved with a $\delta_{e}=6.8^{\circ}$ with working propellers, that the trimmed induced drag is around 8.85 counts. 


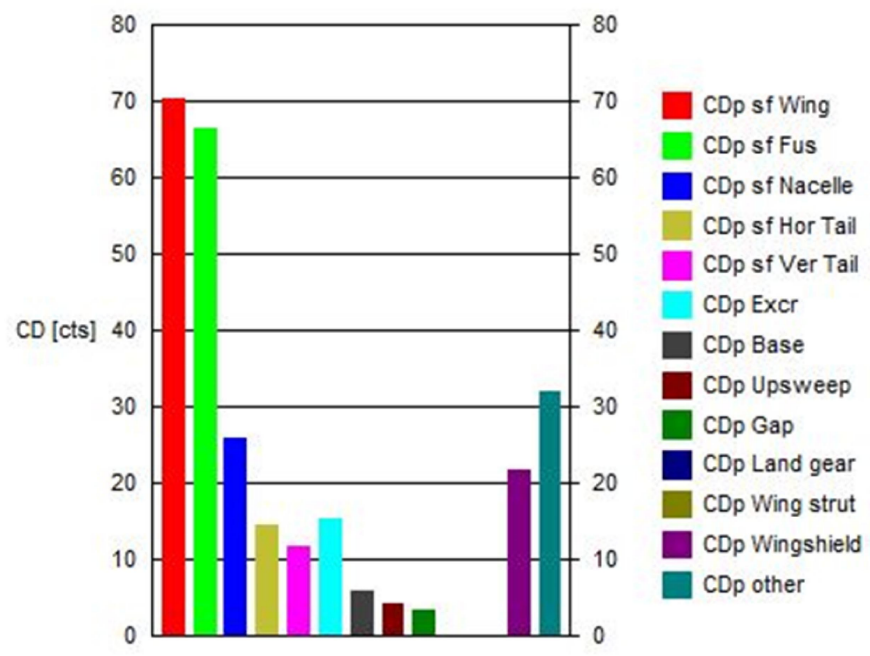

Figure 20. Break down of total estimated parassite drag.

The TWINPROP-16 aircraft has a high-wing with a low horizontal tail where the downwash from the main wing effects the flow field around the tail, which means the efficiency of the horizontal stabilizer may be weakened. The elevator is around $30 \%$ of the chord and extending to full span.

If we take a close look at the contributions of total $C_{L}$ from the components in Figure 29(a), we can see that the horizontal tail is working at an incidence of around $-5.6^{\circ}$, knowing that the tail actual incidence angle $i_{\mathrm{H} 0}=-2^{\circ}$. The downwash effect of the wing can be estimated by

$$
\epsilon_{0}=\frac{2 C_{L, w i n g}}{\pi A R}
$$

From CEASIOM prediction we have $(\mathrm{d} \epsilon / \mathrm{d} \alpha)_{\text {Cruise }}=0.402$ (compared with $(\mathrm{d} \epsilon / \mathrm{d} \alpha)_{\text {Cruise }}=0.343$ from ADAS), it can be calculated that the wing downwash at $\alpha=0^{\circ}$ is $\epsilon \approx-1.94^{\circ}$, resulting in an effective angle of attack for the horizontal tail around $-4^{\circ}$. The fuselage would further effect the horizontal tail. Figure 27 shows the calculations of wing downwash $\epsilon$ and angle of attack effectiveness of the elevator $\tau_{e}$ from Euler solutions for Prop OFF model by assuming the maximum deflected angle $\delta_{e, \max }=-25^{\circ}$.

Figure 28 tracks the streamlines in the Euler solution as they flow over the main wing and continue downstream to the horizontal tail. Notice the negative incidence angle that the horizontal tail is set at $-2^{\circ}$. These streamlines clearly show that the wake of the main wing is washing over the tail. There are no losses in an Euler-simulated wake, but there will be in a real wake, and this will impact the effectiveness of the tail. The streamlines in Fig. 28 are for a low-lift condition $(\alpha=0)$, and consequently show only small downwash angle.

Figure 29(b),(c),(d) show the $C_{m}$ built-up by components from CEASIOM Euler predictions (with propellers off) compared with ADAS semi-empirical results. Both softwares give similar estimation of the main wing and the total configuration with prop-off. The derivative $C_{m, \alpha}$ of the fuselage and nacelles predicted in ADAS and CEASIOM Euler are very close, however the $C_{m, 0}$ (when $\alpha=0$ ) of fuselage and nacelles in Euler calculation is -0.008 compared with 0.008 in ADAS using strip method. Physically correct separation from bodies like the fuselage and nacelle is not accounted for in Euler analysis. The differences therefore are expected due to the semi-empircal method that ADAS uses to account for viscosity, while the viscous effect is not included in CEASIOM Euler analysis. The propeller effect is obvious that it energies the flow behind, and thus increases the horizontal tail efficiency.

The relatively high deviation is in elevator control power $C_{m \delta_{e}}$. The horizontal tail has poor effectiveness since the main wing wake hits the horizontal tail, which is not desirable. This aircraft has a low elevatorchord-to-tail-chord-ratio $c_{e} / c_{\mathrm{H}}=30 \%$, compared to its fellow type Cessna 182 which has $c_{e} / c_{H}=44 \%$. To improve this we need either to design a much larger elevator (increase $c_{e} / c_{\mathrm{H}}$ ) just like Cessna 182, or to lift the stabilizer out of the wake by adopting a T-tail instead. Mounting the propellers might refine the problem thanks to the prop wash. The re-design process can be done in CEASIOM, as Rizzi ${ }^{22}$ did for the 


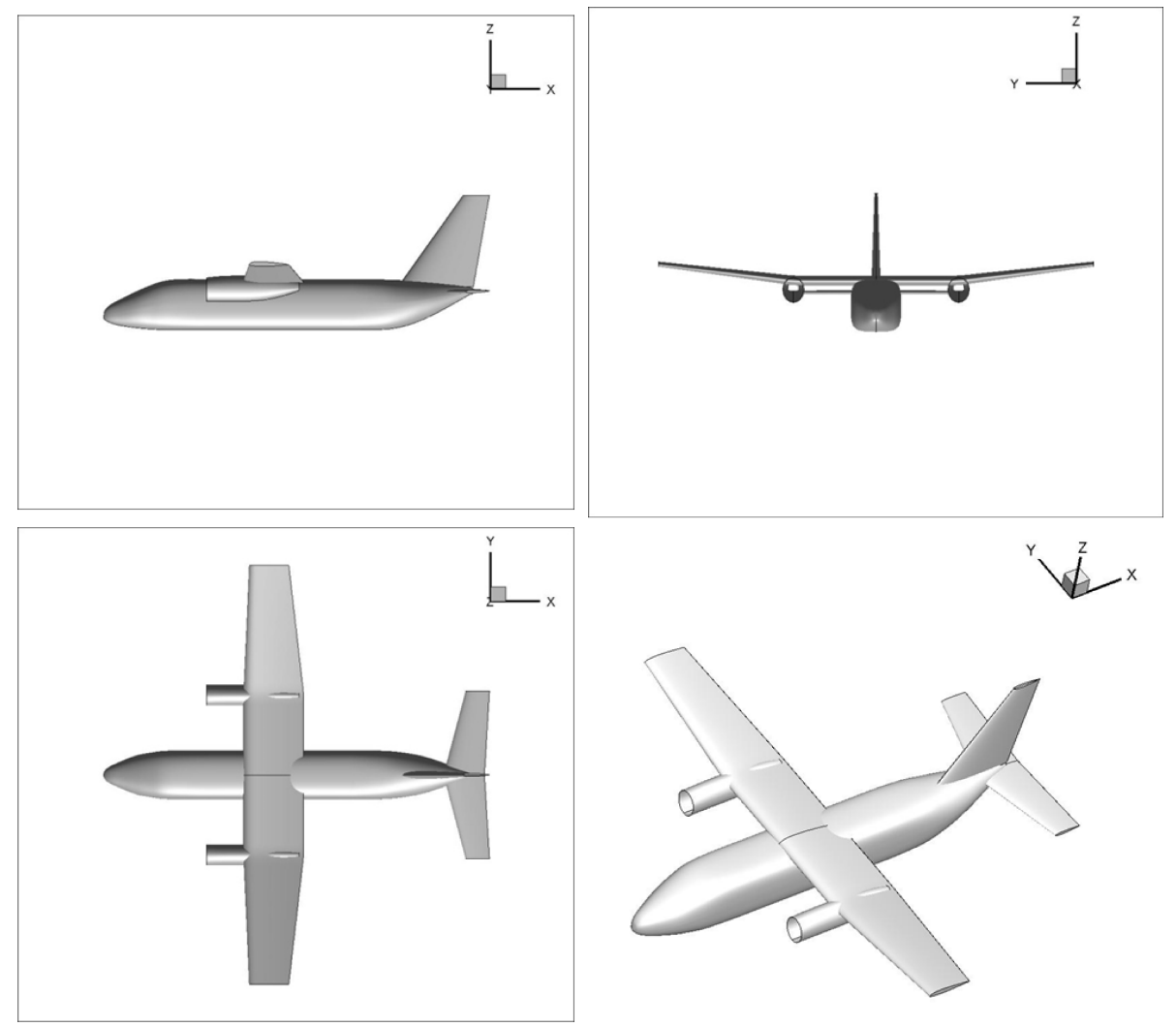

Figure 21. Airplane's external shape.

TransCruiser, a high-speed transport concept.

\section{Conclusions \& Future Work}

The aircraft design stages, conceptual and preliminary, are necessarily collaborative by their very nature. An example design exercise was carried out to illustrate the collaborative aspects of design using using the tools ADAS and CEASIOM, working respectively on conceptual and preliminary design. The example chosen is a FAR-23 compliant 16-seat twin turboprop aircraft for which a high-wing configuration resulted from the ADAS conceptual design. The exercise brought out some of the details involved when exporting the configuration geometry from conceptual design, where the model is usually not water-tight and meshable, to preliminary design where a meshed model is the necessary starting point for further design work. In the example a small computer routine was written to convert the configuration data from ADAS to input data for CEASIOM-sumo so that the configuration was water-tight and meshable. Some manual re-work of the data was required, of the order of several hours (not days!), and such re-work could be further minimized if a standard for the data describing the aircraft would be adopted, for example the CPACS standard proposed by DLR (see $\operatorname{Ref}^{29}$ ).

Euler simulations for 16 flight conditions, some with propeller OFF, and others with propeller ON were computed in order to judge the effect of the propeller wash over the main wing and horizontal tail surface. Within these 16 cases detailed comparisons between ADAS results and CEASIOM results for stability \& control characteristics were carried out. In general there is reasonable agreement between the two sets, considering that the empiricisms in ADAS account for viscous effects where as the CEASIOM are purely inviscid (but nonlinear). The largest discrepancy appears in the pitching moment contribution from the horizontal tail, primarily the elevator control power, and various explanations for this are suggested, including possible effects of the main wing downwash and wake on the tail. Also the empirical models in ADAS predict some lift generated by the fuselage due to viscous separation, where there is none in the Euler results. The propellers can be modeled by Euler in CEASIOM, and the effects are well modeled in inviscid flow and these 


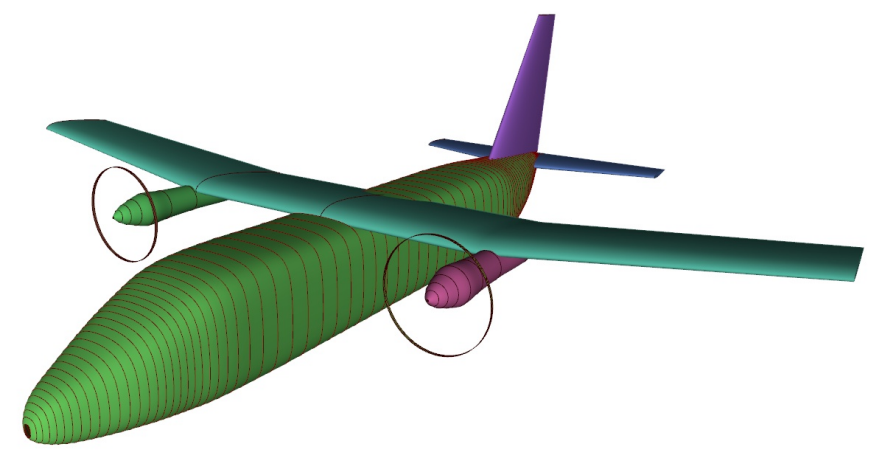

Figure 22. Watertight NURBS model of TWINPROP-16 aircraft created in CEASIOM-sumo with propeller disks

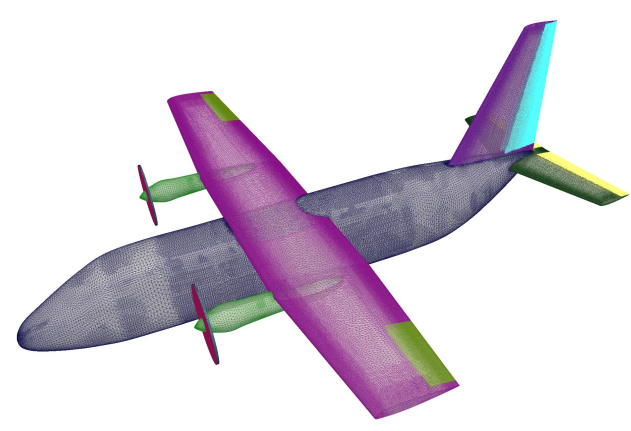

(a) sumo surface mesh

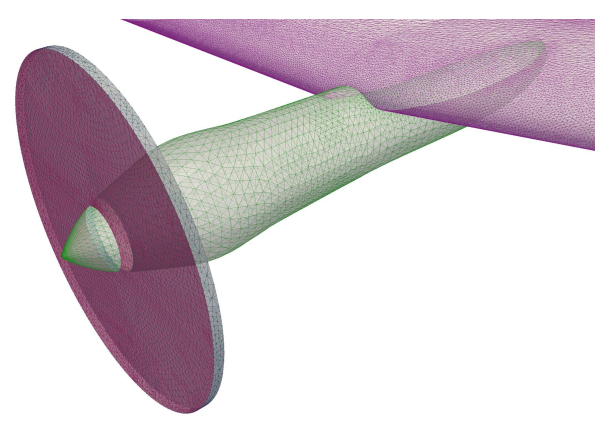

(b) zoom of two-sided propeller disk

Figure 23. TWINPROP-16 aircraft modeled and meshed in CEASIOM-sumo with propellers

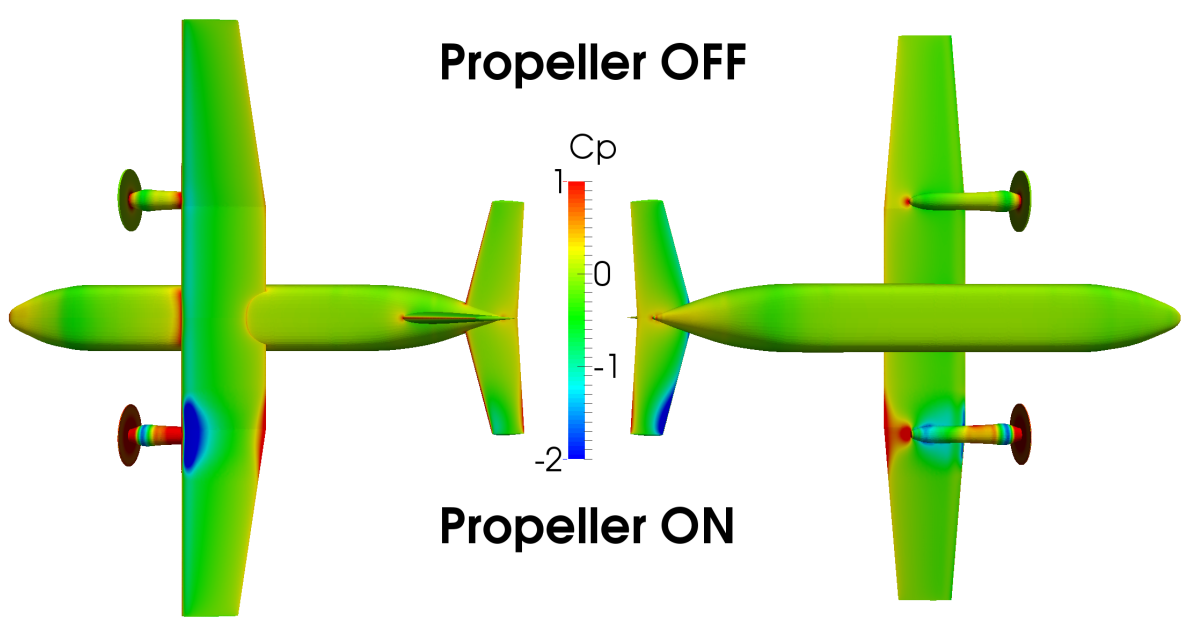

Figure 24. Upper (left) and lower (right) surfaces $C_{P}$ from Euler solutions for TWINPROP-16 aircraft computed in CEASIOM Euler with propellers ON and OFF, Mach $=0.303, \alpha=0^{\circ}, \beta=0^{\circ}$ 

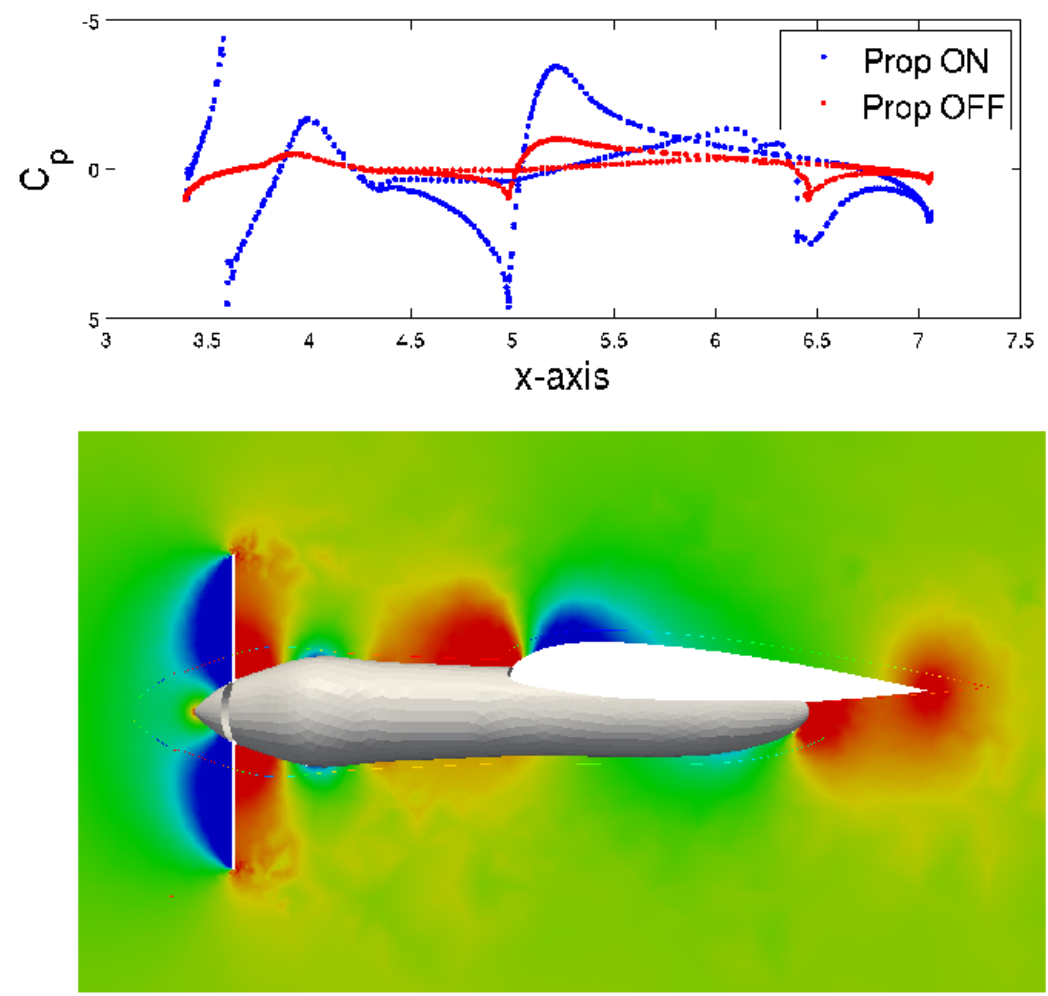

Figure 25. $C_{P}$ in vertical cut through nacelle \& propeller disk, from Euler solutions computed in CEASIOM Euler with propellers ON and OFF, Mach $=0.303, \alpha=0^{\circ}, \beta=0^{\circ}$

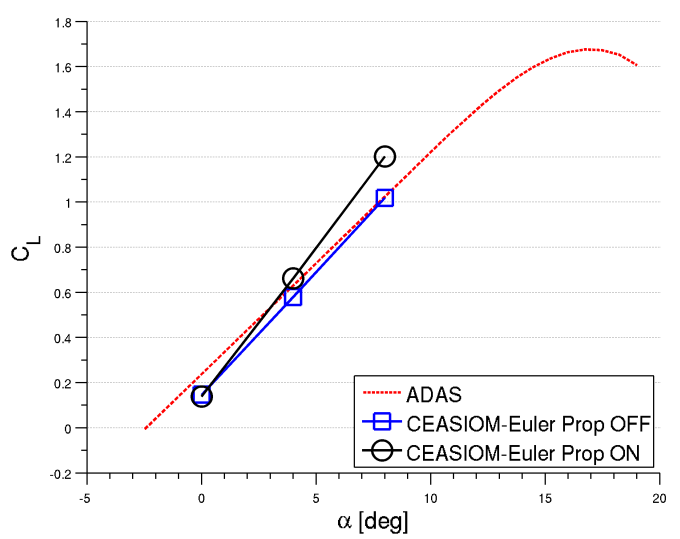

(a) $C_{L}$ vs $\alpha$

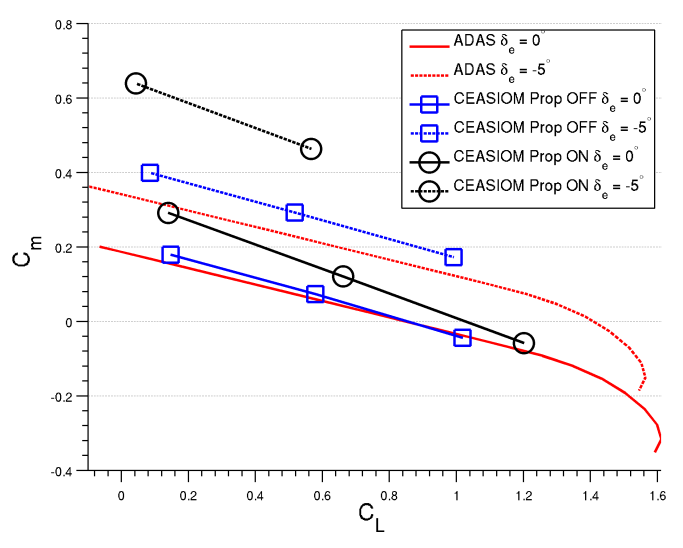

(b) $C_{m}$ vs $C_{L}$

Figure 26. Aerodynamic coefficients from CEASIOM different fidelity solvers for (total) TWINPROP-16 aircraft at $\mathrm{Mach}=0.303$. 
Table 11. Table of solutions for test cases from Euler solver, Mach $=0.303$

\begin{tabular}{cccccccc}
\hline Case no. & $\boldsymbol{\alpha}[\mathrm{deg}]$ & $\boldsymbol{\beta}[\mathrm{deg}]$ & $\boldsymbol{\delta}_{\boldsymbol{r}}[\mathrm{deg}]$ & $\boldsymbol{\delta}_{\boldsymbol{e}}[\mathrm{deg}]$ & $\boldsymbol{\delta}_{\boldsymbol{a}}[\mathrm{deg}]$ & Prop OFF & Prop ON \\
\hline 1 & 0 & 0 & 0 & 0 & 0 & $\mathrm{x}$ & $\mathrm{x}$ \\
2 & 4 & 0 & 0 & 0 & 0 & $\mathrm{x}$ & $\mathrm{x}$ \\
3 & 8 & 0 & 0 & 0 & 0 & $\mathrm{x}$ & $\mathrm{x}$ \\
\hline 4 & 0 & 5 & 0 & 0 & 0 & $\mathrm{x}$ & $\mathrm{x}$ \\
5 & 0 & 10 & 0 & 0 & 0 & $\mathrm{x}$ & $\mathrm{x}$ \\
6 & 8 & 5 & 0 & 0 & 0 & $\mathrm{x}$ & $\mathrm{x}$ \\
7 & 8 & 10 & 0 & 0 & 0 & $\mathrm{x}$ & $\mathrm{x}$ \\
\hline 8 & 0 & 0 & 0 & -5 & 0 & $\mathrm{x}$ & $\mathrm{x}$ \\
9 & 4 & 0 & 0 & -5 & 0 & $\mathrm{x}$ & $\mathrm{x}$ \\
10 & 8 & 0 & 0 & -5 & 0 & $\mathrm{x}$ & $\mathrm{x}$ \\
\hline 11 & 0 & 0 & 5 & 0 & 0 & $\mathrm{x}$ & - \\
12 & 0 & 5 & 5 & 0 & 0 & $\mathrm{x}$ & - \\
13 & 0 & 10 & 5 & 0 & 0 & $\mathrm{x}$ & - \\
\hline 14 & 0 & 0 & 0 & 0 & 5 & $\mathrm{x}$ & - \\
15 & 4 & 0 & 0 & 0 & 5 & $\mathrm{x}$ & - \\
16 & 8 & 0 & 0 & 0 & 5 & $\mathrm{x}$ & - \\
\hline
\end{tabular}

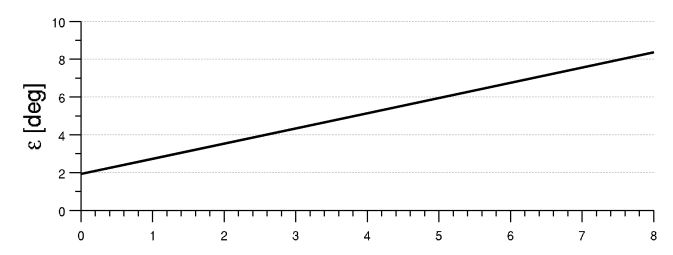

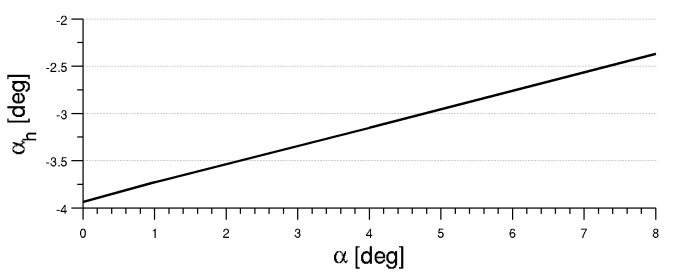

(a) Wing downwash $\epsilon$

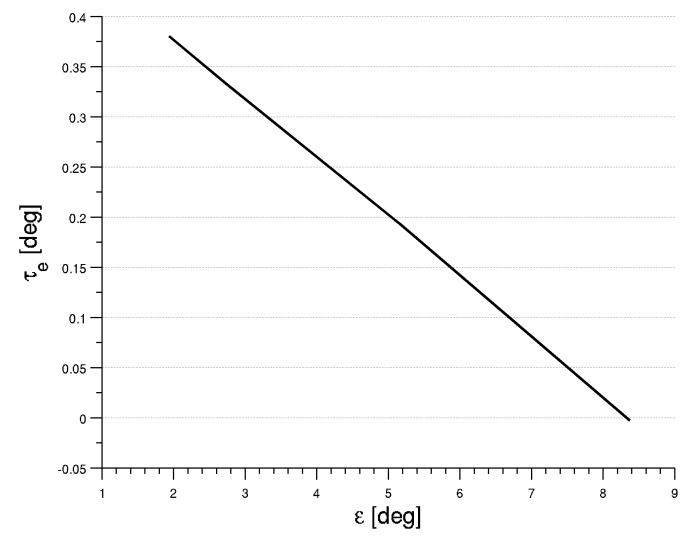

(b) Angle of attack effectiveness of the elevator $\tau_{e}$

Figure 27. Elevator efficiency calculated from CEASIOM Euler solutions for TWINPROP-16 aircraft at Mach $=0.303$.

Table 12. Aerodynamic derivatives

\begin{tabular}{ccccccccc}
\hline Sources & $\boldsymbol{C}_{\boldsymbol{L} \boldsymbol{\alpha}}$ & $\boldsymbol{C}_{\boldsymbol{m} \boldsymbol{\alpha}}$ & $\boldsymbol{C}_{\boldsymbol{Y} \boldsymbol{\beta}}$ & $\boldsymbol{C}_{\boldsymbol{n} \boldsymbol{\beta}}$ & $\boldsymbol{C}_{\boldsymbol{\ell} \boldsymbol{\beta}}$ & $\boldsymbol{C}_{\boldsymbol{m} \boldsymbol{\delta}_{\boldsymbol{e}}}$ & $\boldsymbol{C}_{\boldsymbol{n} \boldsymbol{\delta}_{\boldsymbol{r}}}$ & $\boldsymbol{C}_{\boldsymbol{\ell} \boldsymbol{\delta}_{\boldsymbol{a}}}$ \\
\hline ADAS & 0.102 & -0.0261 & - & 0.0022 & -0.0020 & -0.035 & -0.0031 & - \\
CEASIOM Prop OFF & 0.109 & -0.0278 & -0.0132 & 0.0020 & -0.0015 & -0.042 & -0.0039 & 0.00204 \\
CEASIOM Prop ON & $\mathbf{0 . 1 3 3}$ & $\mathbf{- 0 . 0 4 3 7}$ & -0.0138 & 0.0018 & $\mathbf{- 0 . 0 0 3 4}$ & $\mathbf{- 0 . 0 6 5}$ & - & - \\
\hline
\end{tabular}

Table 13. Break down of contributions to $C_{m}$ prediction from CEASIOM Euler and ADAS for TWINPROP-16 aircraft at $\mathrm{Mach}=\mathbf{0 . 3 0 3}$

\begin{tabular}{ccccccccc}
\hline Sources & $\boldsymbol{C}_{\boldsymbol{m} \mathbf{0}, \mathrm{BNac}}$ & $\boldsymbol{C}_{\boldsymbol{m} \boldsymbol{\alpha}, \mathrm{BNac}}$ & $\boldsymbol{C}_{\boldsymbol{m} \mathbf{0}, \mathrm{W}}$ & $\boldsymbol{C}_{\boldsymbol{m} \boldsymbol{\alpha}, \mathrm{W}}$ & $\boldsymbol{C}_{\boldsymbol{m} \mathbf{0}, \mathrm{H}}$ & $\boldsymbol{C}_{\boldsymbol{m} \boldsymbol{\alpha}, \mathrm{H}}$ & $\boldsymbol{C}_{\boldsymbol{m} \mathbf{0}}$ & $\boldsymbol{C}_{\boldsymbol{m} \boldsymbol{\alpha}}$ \\
\hline ADAS & $\mathbf{0 . 0 0 8}$ & 0.0112 & -0.0121 & -0.00081 & $\mathbf{0 . 1 4 7}$ & -0.0355 & 0.16 & -0.0256 \\
CEASIOM Prop OFF & $\mathbf{- 0 . 0 0 8}$ & 0.0095 & -0.0127 & -0.0011 & $\mathbf{0 . 2 0 7 5}$ & -0.0374 & 0.1789 & -0.0278 \\
CEASIOM Prop ON & - & - & - & - & $\mathbf{0 . 3 2 2}$ & $\mathbf{- 0 . 0 5 3 6}$ & $\mathbf{0 . 2 9 1 7}$ & $\mathbf{- 0 . 0 4 3 7}$ \\
\hline
\end{tabular}




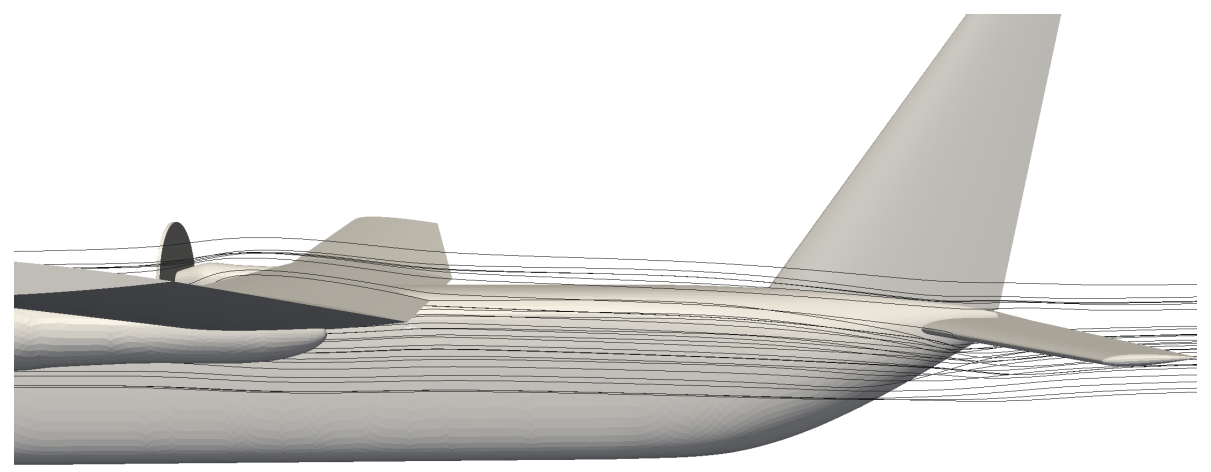

Figure 28. Visualized streamlines around the horizontal tail from CEASIOM Euler solutions for TWINPROP-16 aircraft at $\mathrm{Mach}=\mathbf{0 . 3 0 3}, \alpha=0^{\circ}, \beta=0^{\circ}$

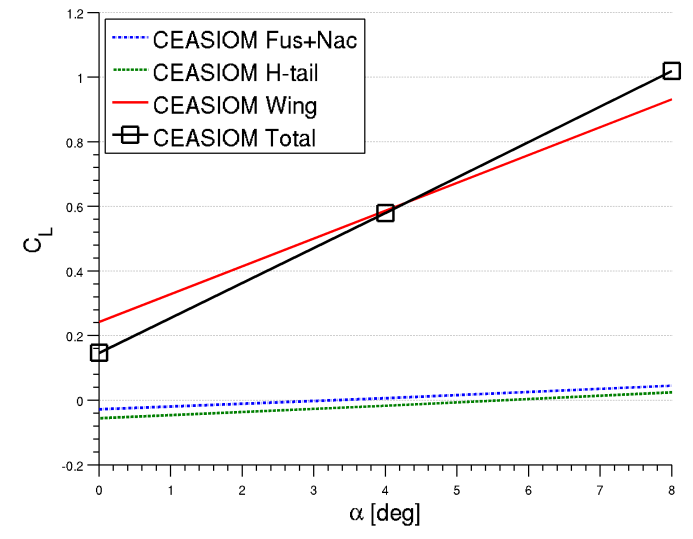

(a) Lift coefficient $C_{L}$ of each component from CEASIOM

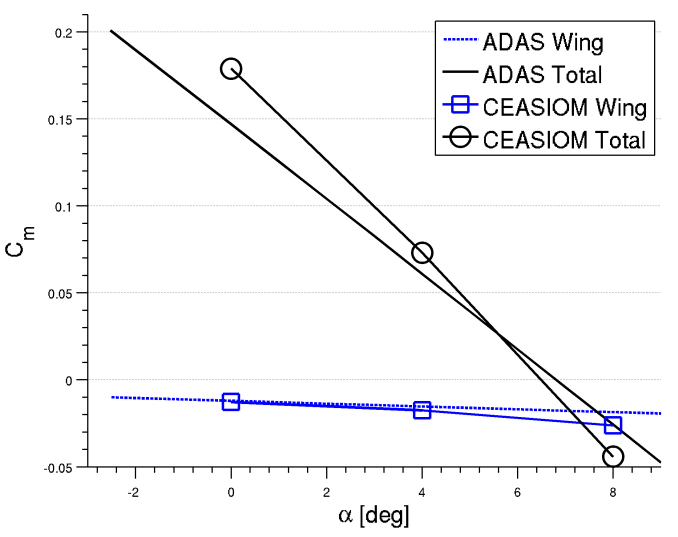

(b) Pitching moment $C_{m}$ of wing and the total aircraft from CEASIOM and ADAS

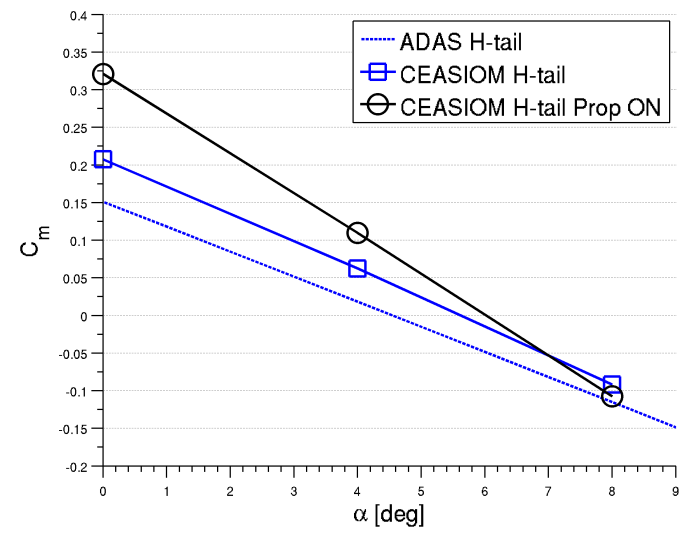

(c) Pitching moment $C_{m}$ of fuselage and nacelle contribu- (d) Pitching moment $C_{m}$ of horizontal tail from CEASIOM tion from CEASIOM and ADAS and ADAS

Figure 29. Break down of contributions to $C_{L}$ (b) $C_{m}$ predicted from CEASIOM Euler solutions and ADAS for TWINPROP-16 aircraft at Mach $=0.303$. 
should be considered in the design process. Note that the propeller effects might be over-estimated by the "finite-thickness" model generated in sumo with mass flow specified. In the future we will make a more realistic model for the propellers, one good example is Lötstedt's work. ${ }^{39}$

This design exercise has pointed out the benefits along with the deficiencies of each of the design tools, highlighting the power of empirical methodology when it applies, together with how physics-based analysis also picks up effects of details that maybe not be included in the empiricism. Obviously no viscous effects are accounted for in an Euler simulation. Therefore future work will be undertaken to revisit this example with RANS analysis where the control surface are individually modeled and meshed for their given deflection angle. For more accurate analysis such like swirl and separation, RANS computations must be made, and will be one of the topics in a future paper. Also a full table of aerodata for the stability and control analysis will be computed to be imported into flight simulation module SDSA. Doing so will provide further perspective on the comparison between empirical-based and physics-based analysis.

\title{
References
}

\begin{abstract}
${ }^{1}$ Nicolosi, F., Paduano, G., "Development of a software for aircraft preliminary design and analysis", 3rd CEAS (Council of European Aerospace Societies) Congress, Venice (Italy), 24-28 October 2011. Proceedings ISBN 978-88-96427-18-7.

${ }^{2}$ Nicolosi, F., Paduano G., "Development of a Software for Aircraft Preliminary Design and Analysis", 10th European Workshop on Aircraft Design Education (EWADE), Dept. Aerospace Engineering, University of Naples Federico II, Naples, Italy, May 2011.
\end{abstract}

${ }^{3}$ Scholz, D., "PreSTo The Aircraft Preliminary Sizing Tool", 10th European Workshop on Aircraft Design Education (EWADE), Dept. Aerospace Engineering, University of Naples Federico II, Naples, Italy, May 2011.

${ }^{4}$ Jenkinson, L. R., Marchman, J. F. III, Aircraft Design Projects for Engineering Students. American Institute of Aeronautics and Astronautics Inc., Reston, USA, 2003.

${ }^{5}$ Coiro D. P., Nicolosi F., "Aircraft Design through Numerical and Experimental Techniques Developed at DPA", Aircraft Design Journal, vol. 4 No. 1 , March, 2001. ISSN 1369-8869.

${ }^{6}$ Coiro D. P., Bellobuono E.F., Nicolosi F., Donelli R., "Improving aircraft Endurance through turbulent separation control by pulsed blowing", Journal of Aircraft, Vol. 45, N. 3, May-June 2008, pp. 990-1001. ISSN 0021-8669, doi: 10.2514/1.33268.

${ }^{7}$ Coiro D. P., Nicolosi F., Scherillo F., Maisto U., "Improving Hang-Glider Maneuverability using multiple winglets: a numerical and experimental investigation", Journal of Aircraft, Vol. 45, N. 3, May-June 2008, pp. 981-989. ISSN 0021-8669, doi: $10.2514 / 1.33265$.

${ }^{8}$ Coiro D. P., Nicolosi F. Grasso F., "Design and Testing of Multi-Element Airfoil for Short-Takeoff-and-Landing Ultralight Aircraft", Journal of Aircraft, Vol. 46, N. 5, September-October 2009, pp. 1795-1807. ISSN 0021-8669, doi: 10.2514/1.43429.

${ }^{9}$ Nicolosi F., De Marco A., Della Vecchia P., "Flight Tests, Performances and Flight Certifcation of a Twin-Engine Light Aircraft", Journal of Aircraft, Vol. 48, N. 1, January-February 2011, pp. 177-192. ISSN 0021-8669, doi: 10.2514/1.C031056.

${ }^{10}$ Nicolosi F., De Marco A., Della Vecchia P., "Stability, Flying Qualities and Longitudinal Parameter Estimation of a Twin-Engine CS23 Certified Light Aircraft", Aerospace Science and Technology, Volume 24, Issue 1, JanuaryFebruary 2013, Pages 226-240. ISSN 1270-9638, http://dx.doi.org/10.1016/j.ast.2012.12.006

${ }^{11}$ Nicolosi F., Della Vecchia P., Ciliberti D., "An investigation on vertical tailplane contribution to aircraft sideforce", Aerospace Science and Technology (Elsevier) AESCTE 2873, Volume 28, Issue 1, July 2013, Pages 401416. ISSN 1270-9638, http://dx.doi.org/10.1016/j.ast.2012.12.006

${ }_{12}$ Nicolosi F., Della Vecchia P., "Aerodynamic Guidelines in the Design and Optimization of new Regional Turboprop Aircraft", 3rd CEAS (Council of European Aerospace Societies) Congress, Venice (Italy), 24-28 October 2011. Proceedings ISBN 978-88-96427-18-7.

${ }^{13}$ Nicolosi F., Della Vecchia P., Corcione S., "Aerodynamic Analysis and Design of a Twin Engine Commuter Aircraft", 28th ICAS (International Council for Aeronautics and Astronautics) Conference, Brisbane (Australia), 23-28 September 2012. Proceedings ISBN 978-0-9565333-1-9 (Optimage Ltd. UK).

${ }^{14}$ Coiro D. P., Nicolosi F., Scherillo F., Maisto U., "Single versus Multiple winglets: Numerical and Experimental Investigation", 26th Congress of the International Council of the Aeronautical Sciences ICAS 2008, Anchorage (Alaska, US), 14-19 September, 2008. ISBN 9781605607153.

${ }^{15}$ Nicolosi, F., Pascale, L., "Design and Aerodyanmic analysis of a light twin-engine propeller aircraft", 26th Congress of the International Council of the Aeronautical Sciences ICAS 2008, Anchorage (Alaska, US), 14-19 September, 2008. ISBN 9781605607153.

${ }^{16}$ Rizzi, A., "Modeling and simulating aircraft stability and control-The SimSAC project", Prog. Aerospace Sci., Vol 47, 2011, pp.573-588. see also www.ceasiom.com (Date 15.12.2011).

${ }^{17}$ Tomac, M. and Eller, D., "From geometry to CFD grids An automated approach for conceptual design", Prog Aerospace Sci, Vol 47, 2011, pp.589596

${ }^{18} \mathrm{Da}$ Ronch, A., Ghoreyshi, M and Badcock, K.J., "On the generation of ight dynamics aerodynamic tables by computational fluid dynamics", Prog Aerospace Sci, Vol 47, 2011, pp.597620

${ }^{19}$ Goetzendorf-Grabowski, T., Mieszalski, D. and Marcinkiewicz, E., "Stability analysis using SDSA tool", Prog AerospaceSci, Vol 47, 2011, pp.636646

${ }^{20}$ Cavagna, L., Ricci., and Travaglini, L., "NeoCASS: An integrated tool for structural sizing, aeroelastic analysis and MDO at conceptual design level", Prog Aerospace Sci, Vol 47, 2011, pp.621635 
${ }^{21}$ Richardson, T.S, Beaverstock, C., Isikveren, A., Meheri, A., Badcock, K.J. and Da Ronch, A., "Analysis of the Boeing 747-100 using CEASIOM", Prog Aerospace Sci, Vol 47, 2011, pp.660673

${ }^{22}$ Rizzi, A., Eliasson, P., Goetzendorf-Grabowski, T., Vos, J.B., Zhang, M. and Richardson, T.S., "Design of a canard configured TransCruiser using CEASIOM", Prog Aerospace Sci, Vol 47, 2011, pp.695705

${ }^{23}$ Tomac, M., "Towards automated CFD for Engineering Methods in Aircraft Design", Doctoral Thesis, KTH Royal Institute of Technology, School of Engineering Sciences, Stockholm, Sweden 2014.

${ }^{24}$ Tomac, M., Eller, D., "Step Towards Automated Robust RANS Meshing", CEAS-0137 4th CEAS Air \& Space Congress, Linkoping, Sweden, Sept 2013.

${ }^{25}$ Baalbergen, E.H., Kos, J. and Lammen, W.F., "Collaborative Multi-Partner Modeling and Simulation Processes to Improve Aeronautical Product Design", 4th CEAS Air \& Space Congress, Linkoping, Sweden, Sept 2013.

${ }^{26}$ Ciampa P.D., Nagel B., Meng, P., Zhang, M., Rizzi A., "Modeling for Physics Based Aircraft Pre-design in a Collaborative Environment", 4th CEAS Air \& Space Conference, Linkoping, Sweden, Sept 2013.

${ }^{27} \mathrm{Si}, \mathrm{H}$., "TetGen: a quality tetrahedral mesh generator and 3D delaunay triangulator", User's Manual, WIAS technical Report No. 13, 2013.

${ }^{28}$ Richardson, T.S, McFarlane, C., Isikveren, A., Badcock, K.J. and Da Ronch, A., "Analysis of conventional and asymmetric aircraft configurations using CEASIOM", Prog Aerospace Sci, Vol 47, 2011, pp.647659

${ }^{29}$ A. Rizzi, M. Zhang, B. Nagel, D. Boehnke, P. Saquet, "Towards a Unified Framework using CPACS for Geometry Management in Aircraft Design", 50th AIAA Aerospace Sciences Meeting, 09-12 January 2012, Nashville, Tennessee

${ }^{30}$ Roskam J., Airplane Design: Part I, Preliminary Sizing of Airplanes, 1st Ed., Roskam Aviation and Engineering Corporation Ottawa, Kansas, 1985.

${ }^{31}$ Anemaat, W. A., Kaushik, B., "Geometry Design Assistant for Airplane Preliminary Design". AIAA-2011-162, 49th AIAA Aerospace Sciences Meeting including the New Horizons Forum and Aerospace Exposition, January 4-7, 2011, Orlando, Florida, USA.

${ }^{32}$ Advanced Aircraft Analysis software, DARcorporation, http://www.darcorp.com/Software/AAA/ (Date 11.11.2013).

${ }^{33}$ Hoak, D. E. et al., "The USAF Stability and Control DATCOM", TR-83-3048, Air Force Wright Aeronautical Laboratories, 1978.

${ }^{34}$ Finck, R. D., "USAF Stability and Control Datcom", AFWAL-TR-83-3048, 1978.

${ }^{35}$ Williams, J. E., Vukelich, S. R., "The USAF Stability and Control Digital DATCOM. Volumes I, II, III. Implementation of Datcom Methods", AFFDL-TR-79-3032, Air Force Wright Aeronautical Laboratories, 1979.

${ }^{36}$ IHS ESDU (Engineering Science Data Unit), ESDU Aerodynamics Series, www.esdu.com (Date 11.11.2013).

${ }^{37}$ Spakovszky, Z. S., "16 Unified: thermodynamics and propulsion", Notes by E. M. Greitzer, Z. S. Spakovszky, I. A. Waitz, 2008.

${ }^{38}$ Rubin, P., "Implementation of a Propeller Model into a Program Solving the Three Dimensional Euler Equations", SAAB Technical Report, 30-May-1991.

${ }^{39}$ Lötstedt, P., "Accuracy of a Propeller Model in Inviscid Flow", Journal of Aircraft, Vol.32, No.6, November-December 1995.

${ }^{40}$ Lamar,J.E., "A Vortex-Lattice Method for the Mean Camber Shapes of Trimmed Non-coplanar Planforms with minimum Vortex Drag", NASA TN D 8090, Washington, D.C., 1976

${ }^{41}$ Nangia, R. K., Palmer, M. E., and Doe, R. H., "Aerodynamic Design Studies of Conventional \& Unconventional Wings with Winglets", AIAA 2000-3400, 2000.

${ }^{42}$ Lyu, Z., Kenway, G. K. W., Martins, J. R. R. A., "RANS-based Aerodynamic Shape Optimization Investigation of the Common Research Model Wing", AIAA SciTech, AIAA 2014-0567.

${ }^{43}$ Etkin, B., Reid, L. D., "Dynamics of Flight: Stablity and Control", 3rd Edition. 